Supporting information to:

\title{
ReaxFF force field development and application for toluene adsorption on MnMOx (M=Cu, Fe, Ni) catalysts
}

\author{
Vjeran Gomzi ${ }^{\mathrm{a}}$, Iva Movre Šapić ${ }^{\mathrm{b}}$, Andrej Vidak \\ vjeran.gomzi@fer.hr
}

${ }^{a}$ Applied physics dept., Faculty of electrical engineering and computing, Unska 3, 10000 Zagreb, Croatia
${ }^{b}$ Physics dept., Faculty of Chemical Engineering and Technology, Marulićev trg 19, 10000 Zagreb, Croatia

Training sets:

$\mathrm{Cu} / \mathrm{Mn} / \mathrm{O} / \mathrm{C} / \mathrm{H}$

COD Id Structure

1526929 CuMn2O4

1530385 CuMn2O4

1533677 Cu1.4Mn1.6O4

$2106773 \mathrm{Cu} 2 \mathrm{Mn} 3 \mathrm{O} 8$

$2227420 \mathrm{C} 10 \mathrm{H} 14 \mathrm{Cu} 2 \mathrm{MnN} 4 \mathrm{O} 14$

4109045 C78 H230Cu17Mn28N12O128

4326303 C14 H26CuMnN2O13

7216595 C3H10Cu0.5Mn0.5O8

$9000847 \mathrm{Cu} 4 \mathrm{H} 14 \mathrm{MnO} 18 \mathrm{~S} 2$

9011507 Cu1.04Mn0.96O2

9011834 CuMnO2

$\mathrm{Fe} / \mathrm{Mn} / \mathrm{O} / \mathrm{C} / \mathrm{H}$

MaterialsProject ID Structure

$\begin{array}{lc}\text { mp-1221857 } & \text { Mn4FeO5 } \\ \text { mp-754642 } & \text { Mn2FeO4 } \\ \text { mp-756562 } & \text { Mn3FeO8 } \\ \text { mp-761563 } & \text { Mn2FeO3 } \\ \text { mp-532236 } & \text { Mn17Fe13O40 } \\ \text { mp-753618 } & \text { Mn9FeO10 } \\ \text { mp-757061 } & \text { Mn4FeO8 } \\ \text { mp-776272 } & \text { Mn5FeO12 } \\ \text { mp-1222437 } & \text { Mn5(FeO3)4 } \\ \text { mp-771188 } & \text { Mn13Fe3O32 } \\ \text { mp-758400 } & \text { Mn5(FeO3)4 } \\ \text { mp-761716 } & \text { MnFe2O3 } \\ \text { mp-38856 } & \text { Mn2FeO4 } \\ \text { mp-754962 } & \text { Mn2FeO6 } \\ \text { mp-674482 } & \text { MnFeO3 } \\ \text { mp-18750 } & \text { Mn(FeO2)2 } \\ \text { mp-1176616 } & \text { Mn3FeO8 } \\ \text { mp-773198 } & \text { Mn5Fe3O16 } \\ \text { mp-1221795 } & \text { MnFe4O5 }\end{array}$




\begin{tabular}{|c|c|}
\hline mp-33708 & $\mathrm{Mn}(\mathrm{FeO} 2) 2$ \\
\hline mp-762538 & Mn13Fe11O32 \\
\hline mp-1221655 & $\mathrm{MnFeO} 4$ \\
\hline mp-769622 & Mn9(FeO6)4 \\
\hline mp-777317 & Mn7Fe3O20 \\
\hline mp-753479 & Mn3Fe3O8 \\
\hline mp-756788 & Mn3FeO8 \\
\hline $\mathrm{mp}-1221648$ & MnFeO2 \\
\hline mp-1221814 & MnFe5O8 \\
\hline mp-755276 & Mn5FeO12 \\
\hline mp-761315 & MnFeO4 \\
\hline mp-771261 & Mn3FeO8 \\
\hline mp-759744 & MnFeO3 \\
\hline $\mathrm{mp}-1221773$ & MnFe2O3 \\
\hline mp-757698 & Mn23FeO32 \\
\hline mp-697842 & $\mathrm{Mn}(\mathrm{FeO} 2) 2$ \\
\hline mp-757662 & Mn3Fe5O12 \\
\hline mp-753787 & Mn2FeO6 \\
\hline mp-752751 & Mn2FeO6 \\
\hline mp-706492 & Mn19Fe17O48 \\
\hline
\end{tabular}

$\mathrm{Ni} / \mathrm{Mn} / \mathrm{O} / \mathrm{C} / \mathrm{H}$

COD Id Structure

$1530384 \mathrm{Mn} 2 \mathrm{NiO} 4$

1541565 MnNi0.5O4V1.5

4118052 C33H24Mn0.5Ni1.5O16

$5910161 \mathrm{Mn} 2 \mathrm{NiO} 4$

7115434 C68H140Mn24Ni2O84 7203362 C4H6Mn0.79Ni0.21O6

9013975 MnNi6O8

9013976 Mn0.8Ni6.4O8

9013977 Mn0.6Ni6.8O8

9013978 Mn0.4Ni7.2O8

9013979 Mn0.2Ni7.6O8 


\section{$\mathrm{Cu} / \mathrm{Mn} / \mathrm{O} / \mathrm{C} / \mathrm{H}$ Force field}

Refinement of:

$\mathrm{Cu}$ : Reactive Dynamics Simulation of Monolayer and Multilayer Adsorption of Glycine on Cu(110) Susanna Monti*a, Cui Li b and Vincenzo Carravetta b

Mn: Electrode-electrolyte interface layers in lithium ion batteries using reactive force field based molecular dynamics by Sahithya Reddivari, doctoral thesis, 2016, University of Michigan

Reactive MD-force field: Mn/Cu/O/C/H/N Gomzi et al 2021.

39 ! Number of general parameters

50.0000 !Overcoordination parameter

9.5469 !Overcoordination parameter

1.6725 !Valency angle conjugation parameter

1.7224 !Triple bond stabilisation parameter

6.8702 !Triple bond stabilisation parameter

60.4850 !C2-correction

1.0588 !Undercoordination parameter

4.6000 !Triple bond stabilisation parameter

12.1176 !Undercoordination parameter

13.3056 !Undercoordination parameter

-55.1978 !Triple bond stabilization energy

0.0000 !Lower Taper-radius

10.0000 !Upper Taper-radius

2.8793 !Not used

33.8667 !Valency undercoordination

6.0891 !Valency angle/lone pair parameter

1.0563 !Valency angle

2.0384 !Valency angle parameter

6.1431 !Not used

6.9290 !Double bond/angle parameter

0.3989 !Double bond/angle parameter: overcoord

3.9954 !Double bond/angle parameter: overcoord

-2.4837 !Not used

5.7796 !Torsion/BO parameter

10.0000 !Torsion overcoordination

1.9487 !Torsion overcoordination

-1.2327 !Conjugation 0 (not used)

2.1645 !Conjugation

1.5591 !vdWaals shielding

0.1000 !Cutoff for bond order $(* 100)$

1.7602 !Valency angle conjugation parameter

0.6991 !Overcoordination parameter

50.0000 !Overcoordination parameter

1.8512 !Valency/lone pair parameter

0.5000 !Not used

20.0000 !Not used

5.0000 !Molecular energy (not used)

0.0000 !Molecular energy (not used)

0.7903 !Valency angle conjugation parameter

12 ! Nr of atoms; cov.r; valency;a.m;Rvdw;Evdw;gammaEEM;cov.r2;

alfa;gammavdW;valency;Eunder;Eover;chiEEM;etaEEM;n.u.

cov r3;Elp;Heat inc.;n.u.;n.u.;n.u.;n.u. 
ov/un;val1;n.u.;val3,vval4

C $\quad \begin{array}{llllllll}1.3817 & 4.0000 & 12.0000 & 1.8903 & 0.1838 & 0.6387 & 1.1341 & 4.0000\end{array}$ $\begin{array}{llllllll}9.7559 & 2.1346 & 4.0000 & 34.9350 & 79.5548 & 4.9218 & 6.0000 & 0.0000\end{array}$

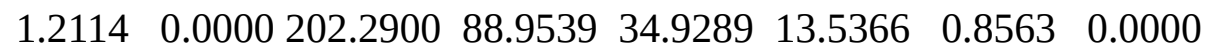
$\begin{array}{llllllll}-2.8983 & 2.5000 & 1.0560 & 44.0000 & 2.9663 & 0.0000 & 0.0000 & 0.0000\end{array}$

$\mathrm{H} \quad \begin{array}{lllllllll}\mathrm{H} & 0.8930 & 1.0000 & 1.0080 & 1.3550 & 0.0930 & 0.8203 & -0.1000 & 1.0000\end{array}$ $\begin{array}{llllllll}8.2230 & 33.2894 & 1.0000 & 0.0000 & 121.1250 & 3.7248 & 9.6093 & 1.0000\end{array}$ $\begin{array}{llllllll}-0.1000 & 0.0000 & 55.1870 & 83.0408 & 2.4197 & 0.0003 & 1.0698 & 0.0000\end{array}$ $\begin{array}{llllllll}-19.4571 & 4.2733 & 1.0330 & 81.0000 & 2.8793 & 0.0000 & 0.0000 & 0.0000\end{array}$

$\begin{array}{lllllllll}\text { O } & 1.2450 & 2.0000 & 15.9990 & 2.3890 & 0.1000 & 1.0898 & 1.0548 & 6.0000\end{array}$ $\begin{array}{llllllll}9.7300 & 13.8449 & 4.0000 & 37.5000 & 116.0768 & 8.5000 & 8.3122 & 2.0000\end{array}$ $\begin{array}{llllllll}0.9049 & 0.4056 & 68.0152 & 3.5027 & 0.7640 & 0.0021 & 0.9745 & 0.0000\end{array}$ $\begin{array}{llllllll}-3.5500 & 2.9000 & 1.0493 & 4.0000 & 2.9225 & 0.0000 & 0.0000 & 0.0000\end{array}$ $\begin{array}{llllllll}\text { Mn } 2.2190 & 7.0000 & 54.9380 & 1.9685 & 0.3855 & 0.4633 & 0.1000 & 6.0000\end{array}$ $\begin{array}{lllllllll}11.2058 & 4.1928 & 4.0000 & 0.0000 & 0.0000 & -1.0000 & 6.1911 & 0.0000\end{array}$

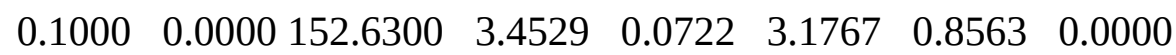
$\begin{array}{lllllllll}-25.0000 & 3.1072 & 1.0338 & 8.0000 & 3.4590 & 0.0000 & 0.0000 & 0.0000\end{array}$

$\begin{array}{lllllllll}\mathrm{Li} & 1.9814 & 1.0000 & 6.9410 & 1.8000 & 0.2939 & 0.9387 & -0.1000 & 1.0000\end{array}$ $\begin{array}{llllllll}9.0616 & 1.3258 & 1.0000 & 0.0000 & 0.0000 & -3.0000 & 10.0241 & 0.0000\end{array}$ $\begin{array}{llllllll}-1.0000 & 0.0000 & 37.5000 & 5.4409 & 6.9107 & 0.1973 & 0.8563 & 0.0000\end{array}$ $\begin{array}{llllllll}-2.5068 & 2.2989 & 1.0338 & 1.0000 & 2.8103 & 1.3000 & 0.2000 & 13.0000\end{array}$

$\begin{array}{llllllllll}\text { F } & 1.7938 & 1.0000 & 18.9984 & 1.4139 & 0.3134 & 0.7750 & -0.1000 & 7.0000\end{array}$ $\begin{array}{llllllll}10.3051 & 15.1397 & 1.0000 & 9.2533 & 0.2000 & 9.8105 & 8.6941 & 0.0000\end{array}$ $\begin{array}{llllllll}-1.0000 & 3.5571 & 18.0000 & 6.9821 & 4.1799 & 1.0561 & 0.0000 & 0.0000\end{array}$ $\begin{array}{llllllll}-6.3417 & 2.6656 & 1.0493 & 4.0000 & 2.9225 & 0.0000 & 0.0000 & 0.0000\end{array}$

$\begin{array}{lllllllll}\mathrm{P} & 1.5994 & 3.0000 & 30.9738 & 1.7000 & 0.1743 & 1.0385 & 1.3000 & 5.0000\end{array}$ $\begin{array}{llllllll}9.1909 & 14.2932 & 5.0000 & 0.0000 & 0.0000 & 0.9528 & 7.9121 & 0.0000\end{array}$ $\begin{array}{llllllll}-1.0000 & 10.2596 & 1.5000 & 0.2205 & 16.7429 & 15.9629 & 0.0000 & 0.0000\end{array}$ $\begin{array}{llllllll}-2.5000 & 1.6114 & 1.0338 & 5.0000 & 2.8793 & 0.0000 & 0.0000 & 0.0000\end{array}$

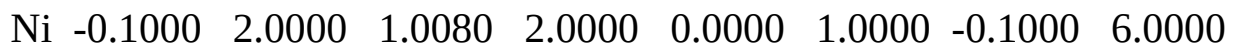
$\begin{array}{llllllll}10.0000 & 2.5000 & 4.0000 & 0.0000 & 0.0000 & 8.5000 & 1.5000 & 0.0000\end{array}$ $\begin{array}{lllllllll}-0.1000 & 0.0000 & -2.3700 & 8.7410 & 13.3640 & 0.6690 & 0.9745 & 0.0000\end{array}$ $\begin{array}{lllllllll}-11.0000 & 2.7466 & 1.0338 & 6.2998 & 2.8793 & 0.0000 & 0.0000 & 0.0000\end{array}$

$\begin{array}{lllllllll}\text { Al } & -0.1000 & 2.0000 & 1.0080 & 2.0000 & 0.0000 & 1.0000 & -0.1000 & 6.0000\end{array}$ $\begin{array}{llllllll}10.0000 & 2.5000 & 4.0000 & 0.0000 & 0.0000 & 8.5000 & 1.5000 & 0.0000\end{array}$ $\begin{array}{llllllll}-0.1000 & 0.0000 & -2.3700 & 8.7410 & 13.3640 & 0.6690 & 0.9745 & 0.0000\end{array}$ $\begin{array}{llllllll}-11.0000 & 2.7466 & 1.0338 & 6.2998 & 2.8793 & 0.0000 & 0.0000 & 0.0000\end{array}$

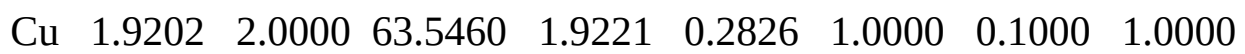
$\begin{array}{llllllll}10.9889 & 100.0000 & 1.0000 & 0.0000 & 0.0000 & 2.7875 & 6.0000 & 0.0000\end{array}$ $\begin{array}{llllllll}-1.0000 & 0.0000 & 80.7000 & 34.9555 & 0.4988 & 0.0000 & 0.8563 & 0.0000\end{array}$ $\begin{array}{llllllll}-5.1872 & 3.1491 & 1.0000 & 4.0000 & 2.5791 & 0.0000 & 0.0000 & 0.0000\end{array}$

N $\quad \begin{array}{llllllll}1.2333 & 3.0000 & 14.0000 & 2.1263 & 0.1207 & 1.0000 & 1.1748 & 5.0000\end{array}$ $\begin{array}{llllllll}9.9865 & 13.2428 & 4.0000 & 26.4087 & 100.0000 & 6.3619 & 6.9188 & 2.0000\end{array}$

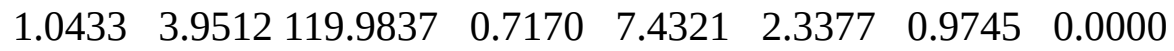
$\begin{array}{llllllll}-3.5800 & 4.0000 & 1.0183 & 4.0000 & 2.8793 & 0.0000 & 0.0000 & 0.0000\end{array}$

S $\quad \begin{array}{llllllll}1.9673 & 2.0000 & 32.0600 & 2.1729 & 0.3000 & 1.0336 & 1.5359 & 6.0000\end{array}$ $\begin{array}{llllllll}10.3008 & 4.9055 & 4.0000 & 52.9998 & 112.1416 & 6.5000 & 8.2545 & 2.0000\end{array}$ $\begin{array}{llllllll}1.4601 & 9.7177 & 71.1843 & 5.7487 & 23.2859 & 12.7147 & 0.9745 & 0.0000\end{array}$ $\begin{array}{llllllll}-11.0000 & 2.7466 & 1.0338 & 6.2998 & 2.8793 & 0.0000 & 0.0000 & 0.0000\end{array}$

$42 \quad$ ! Nr of bonds; Edis1;LPpen;n.u.;pbe1;pbo5;13corr;pbo6 pbe2;pbo3;pbo4;Etrip;pbo1;pbo2;ovcorr

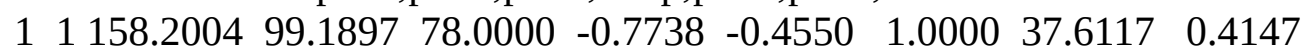


$\begin{array}{llllllll}0.4590 & -0.1000 & 9.1628 & 1.0000 & -0.0777 & 6.7268 & 1.0000 & 0.0000\end{array}$

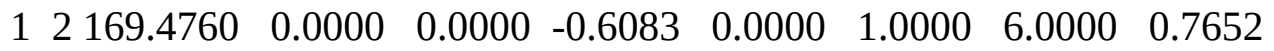
$\begin{array}{llllllll}5.2290 & 1.0000 & 0.0000 & 1.0000 & -0.0553 & 6.9316 & 0.0000 & 0.0000\end{array}$

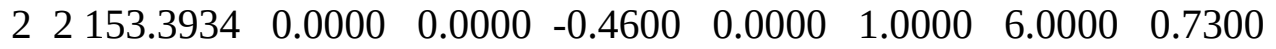
$\begin{array}{llllllll}6.2500 & 1.0000 & 0.0000 & 1.0000 & -0.0790 & 6.0552 & 0.0000 & 0.0000\end{array}$

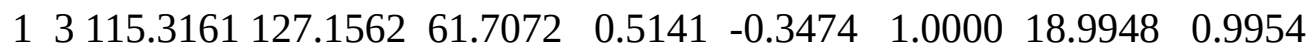
$\begin{array}{llllllll}1.5618 & -0.3414 & 8.9489 & 1.0000 & -0.1628 & 5.6821 & 0.0000 & 0.0000\end{array}$

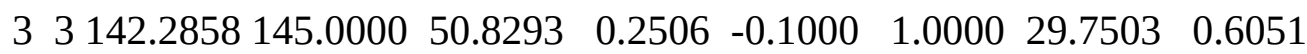
$\begin{array}{llllllll}0.3451 & -0.1055 & 9.0000 & 1.0000 & -0.1225 & 5.5000 & 1.0000 & 0.0000\end{array}$

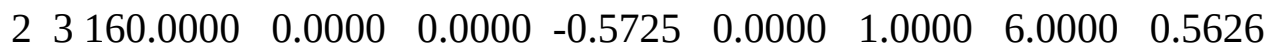
$\begin{array}{llllllll}1.1150 & 1.0000 & 0.0000 & 0.0000 & -0.0920 & 4.2790 & 0.0000 & 0.0000\end{array}$

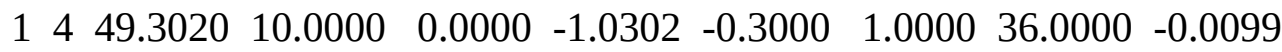
$\begin{array}{llllllll}1.8910 & -0.4204 & 7.6871 & 1.0000 & -0.0880 & 6.6676 & 1.0000 & 0.0000\end{array}$

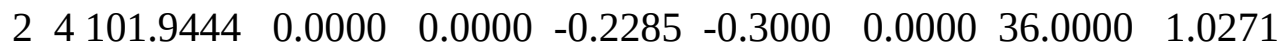
$\begin{array}{llllllll}7.0832 & -0.1532 & 19.8594 & 1.0000 & -0.0836 & 4.7210 & 0.0000 & 0.0000\end{array}$

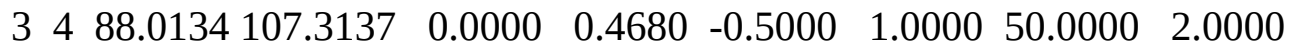
$\begin{array}{llllllll}7.5815 & -0.4509 & 7.6953 & 1.0000 & -0.0952 & 5.1424 & 1.0000 & 0.0000\end{array}$

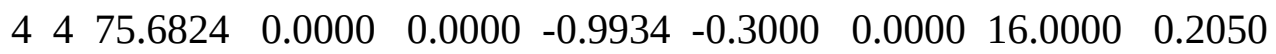
$\begin{array}{llllllll}2.2857 & -0.3000 & 16.0000 & 1.0000 & -0.1230 & 8.7005 & 0.0000 & 0.0000\end{array}$

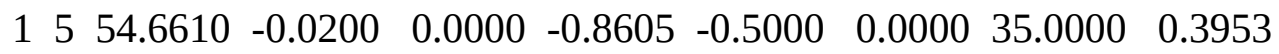
$\begin{array}{llllllll}0.6908 & -0.2500 & 11.9965 & 1.0000 & -0.0668 & 9.0596 & 0.0000 & 0.0000\end{array}$

$\begin{array}{llllllllll}2 & 5 & 59.2034 & 0.0000 & 0.0000 & 0.1240 & 0.0000 & 0.0000 & 6.0000 & 0.4000\end{array}$ $\begin{array}{llllllll}1.0000 & 0.0000 & 12.0000 & 1.0000 & -0.0565 & 4.9575 & 0.0000 & 0.0000\end{array}$

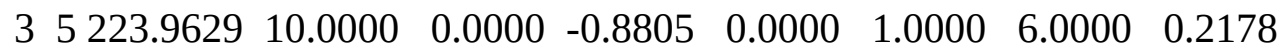
$\begin{array}{llllllll}4.2326 & -0.1048 & 7.0167 & 1.0000 & -0.0867 & 4.2825 & 0.0000 & 0.0000\end{array}$

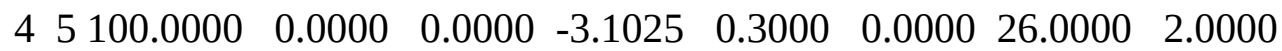
$\begin{array}{llllllll}3.2823 & 0.0000 & 12.0000 & 1.0000 & -0.0800 & 6.9752 & 0.0000 & 0.0000\end{array}$

$\begin{array}{llllllllll}5 & 5 & 34.3154 & 0.0000 & 0.0000 & 0.5995 & 0.3000 & 0.0000 & 26.0000 & 0.5445\end{array}$ $\begin{array}{llllllll}0.5752 & 0.0000 & 12.0000 & 1.0000 & -0.1382 & 4.5000 & 0.0000 & 0.0000\end{array}$

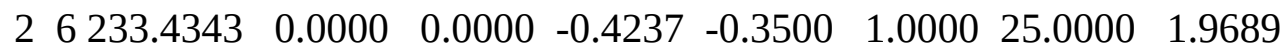
$\begin{array}{llllllll}4.7870 & -0.2500 & 15.0000 & 1.0000 & -0.1288 & 4.9159 & 1.0000 & 0.0000\end{array}$

$\begin{array}{llllllllll}3 & 6 & 197.9420 & 0.0000 & 0.0000 & -0.7552 & -0.3500 & 1.0000 & 25.0000 & 0.2137\end{array}$ $\begin{array}{llllllll}9.5888 & -0.2500 & 15.0000 & 1.0000 & -0.1477 & 7.8947 & 1.0000 & 0.0000\end{array}$

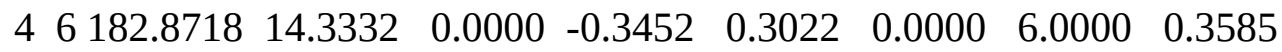
$\begin{array}{llllllll}2.6684 & -0.1484 & 11.8165 & 1.0000 & -0.0940 & 5.8491 & 0.0000 & 0.0000\end{array}$

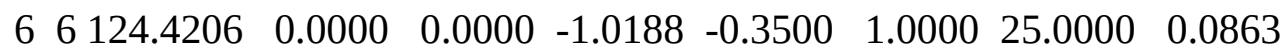
$\begin{array}{llllllll}8.4257 & -0.2500 & 15.0000 & 1.0000 & -0.2337 & 8.1452 & 1.0000 & 0.0000\end{array}$

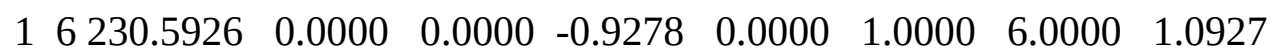
$\begin{array}{llllllll}7.1644 & 1.0000 & 0.0000 & 1.0000 & -0.0732 & 5.9745 & 0.0000 & 0.0000\end{array}$

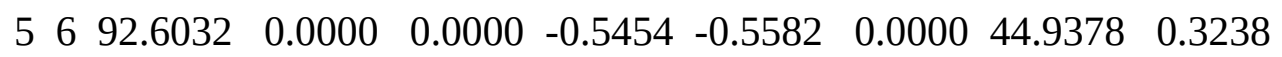
$\begin{array}{llllllll}1.0563 & -0.2500 & 15.0000 & 1.0000 & -0.1206 & 4.0000 & 0.0000 & 0.0000\end{array}$

$\begin{array}{lllllllll}1 & 7110.0000 & 92.0000 & 0.0000 & 0.2171 & -0.1418 & 1.0000 & 13.1260 & 0.6000\end{array}$ $\begin{array}{llllllll}0.3601 & -0.1310 & 10.7257 & 1.0000 & -0.0869 & 5.3302 & 1.0000 & 0.0000\end{array}$

$\begin{array}{llllllllll}2 & 7 & 0.1466 & 0.0000 & 0.0000 & 0.2250 & -0.1418 & 1.0000 & 13.1260 & 0.6000\end{array}$ $\begin{array}{llllllll}0.3912 & -0.1310 & 0.0000 & 1.0000 & -0.1029 & 9.3302 & 0.0000 & 0.0000\end{array}$

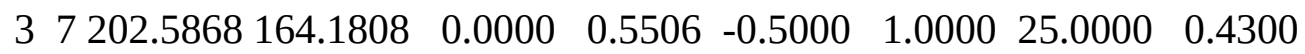
$\begin{array}{llllllll}0.0912 & -0.1285 & 16.0342 & 1.0000 & -0.2008 & 6.2678 & 1.0000 & 0.0000\end{array}$

$\begin{array}{llllllllll}7 & 7 & 0.0000 & 0.0000 & 0.0000 & 0.2171 & -0.5000 & 1.0000 & 35.0000 & 0.6000\end{array}$ $\begin{array}{llllllll}0.5000 & -0.5000 & 20.0000 & 1.0000 & -0.2000 & 10.0000 & 1.0000 & 0.0000\end{array}$

$\begin{array}{lllllllll}6 & 7191.3390 & 10.2597 & 0.0000 & 0.9085 & -0.5000 & 1.0000 & 0.0000 & 0.1616\end{array}$ $\begin{array}{llllllll}0.0940 & -0.1039 & 10.0002 & 1.0000 & -0.1073 & 9.9960 & 1.0000 & 0.0000\end{array}$

$\begin{array}{llllllllll}5 & 7 & 0.0000 & 0.0000 & 0.0000 & 0.5000 & -0.2000 & 0.0000 & 16.0000 & 0.5000\end{array}$ 
$\begin{array}{llllllll}1.0001 & -0.2000 & 15.0000 & 1.0000 & -0.1000 & 10.0000 & 0.0000 & 0.0000\end{array}$

$\begin{array}{llllllllll}4 & 7 & 0.0000 & 0.0000 & 0.0000 & 0.5000 & -0.2000 & 0.0000 & 16.0000 & 0.5000\end{array}$

$\begin{array}{llllllll}1.0001 & -0.2000 & 15.0000 & 1.0000 & -0.1000 & 10.0000 & 0.0000 & 0.0000\end{array}$

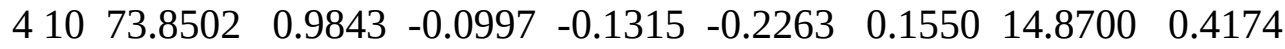

$\begin{array}{llllllll}0.3101 & -0.2125 & 14.9850 & 1.0420 & -0.1266 & 6.0687 & -0.5253 & 0.4960\end{array}$

$\begin{array}{lllllllll}110 & 0.0000 & 0.0000 & 0.0000 & 0.2000 & -0.1418 & 1.0000 & 13.1260 & 0.5000\end{array}$

$\begin{array}{llllllll}0.5000 & -0.2000 & 20.0000 & 1.0000 & -0.1000 & 9.0000 & 0.0000 & 0.0000\end{array}$

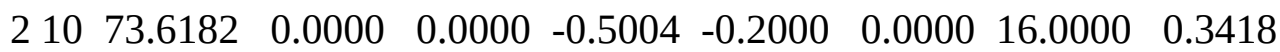

$\begin{array}{llllllll}9.0000 & -0.2000 & 15.0000 & 1.0000 & -0.1015 & 5.7850 & 0.0000 & 0.0000\end{array}$

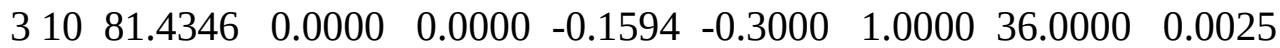

$\begin{array}{llllllll}0.2904 & -0.2500 & 12.0000 & 1.0000 & -0.0742 & 9.3638 & 0.0000 & 0.0000\end{array}$

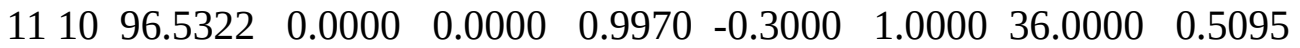

$\begin{array}{llllllll}0.7247 & -0.2500 & 12.0000 & 1.0000 & -0.1175 & 9.9985 & 0.0000 & 0.0000\end{array}$

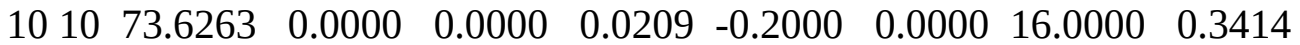

$\begin{array}{llllllll}0.4703 & -0.2000 & 15.0000 & 1.0000 & -0.1319 & 5.9254 & 0.0000 & 0.0000\end{array}$

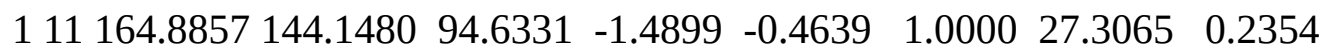

$\begin{array}{llllllll}0.3170 & -0.3518 & 7.1266 & 1.0000 & -0.2521 & 4.5741 & 1.0000 & 0.0000\end{array}$

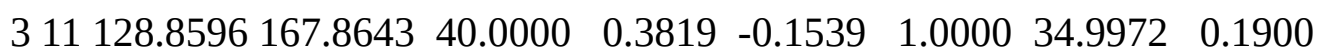

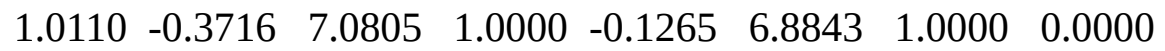

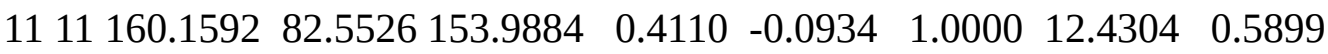

$\begin{array}{llllllll}0.1538 & -0.1473 & 11.9187 & 1.0000 & -0.0753 & 5.4371 & 1.0000 & 0.0000\end{array}$

$\begin{array}{lllllllll}211 & 211.0748 & 0.0000 & 0.0000 & -0.3810 & 0.0000 & 1.0000 & 6.0000 & 0.4878\end{array}$

$\begin{array}{llllllll}2.7875 & 1.0000 & 0.0000 & 1.0000 & -0.1608 & 5.6332 & 0.0000 & 0.0000\end{array}$

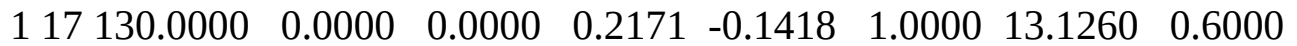

$\begin{array}{llllllll}0.3601 & -0.1310 & 10.7257 & 1.0000 & -0.0869 & 5.3302 & 1.0000 & 0.0000\end{array}$

$\begin{array}{lllllllll}411 & 0.0000 & 0.0000 & 0.0000 & 0.5000 & -0.2000 & 0.0000 & 16.0000 & 0.5000\end{array}$

$\begin{array}{llllllll}1.0001 & -0.2000 & 15.0000 & 1.0000 & -0.1000 & 10.0000 & 0.0000 & 0.0000\end{array}$

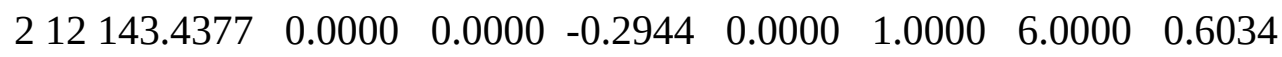

$\begin{array}{llllllll}9.5627 & 1.0000 & 0.0000 & 1.0000 & -0.0516 & 7.0960 & 1.0000 & 0.0000\end{array}$

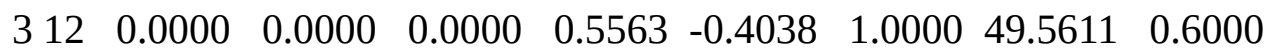

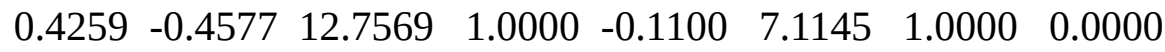

26 ! Nr of off-diagonal terms; Ediss;Ro;gamma;rsigma;rpi;rpi2

$\begin{array}{llllllll}1 & 2 & 0.1239 & 1.4004 & 9.8467 & 1.1210 & -1.0000 & -1.0000\end{array}$

$\begin{array}{llllllll}2 & 3 & 0.0283 & 1.2885 & 10.9190 & 0.9215 & -1.0000 & -1.0000\end{array}$

$\begin{array}{llllllll}1 & 3 & 0.0503 & 1.8006 & 10.2114 & 1.3492 & 1.1992 & 1.0506\end{array}$

$\begin{array}{llllllll}1 & 4 & 0.0475 & 1.6810 & 8.8902 & 1.5153 & 1.0000 & -1.0000\end{array}$

$\begin{array}{llllllll}2 & 4 & 0.1017 & 1.3259 & 12.5406 & 1.4534 & -1.0000 & -1.0000\end{array}$

$\begin{array}{llllllll}3 & 4 & 0.1121 & 2.0879 & 11.2110 & 1.5063 & 1.4795 & -1.0000\end{array}$

$\begin{array}{llllllll}1 & 5 & 0.0270 & 2.4124 & 11.4640 & 1.7840 & 1.0000 & 1.0000\end{array}$

$\begin{array}{llllllll}2 & 5 & 0.1149 & 1.4658 & 11.0886 & 1.3337 & -1.0000 & -1.0000\end{array}$

$\begin{array}{llllllll}3 & 5 & 0.0687 & 1.8765 & 12.0451 & 1.2928 & -1.0000 & -1.0000\end{array}$

$\begin{array}{llllllll}4 & 5 & 7.1011 & 0.9961 & 40.6852 & 1.7459 & -1.0000 & -1.0000\end{array}$

$\begin{array}{lllllllll}2 & 6 & 0.1087 & 1.8734 & 9.1166 & 1.0000 & -1.0000 & -1.0000\end{array}$

$\begin{array}{llllllll}3 & 6 & 0.1714 & 1.6576 & 10.7298 & 1.5553 & -1.0000 & -1.0000\end{array}$

$\begin{array}{llllllll}4 & 6 & 0.0837 & 1.9512 & 12.2762 & 1.4795 & -1.0000 & -1.0000\end{array}$

$\begin{array}{llllllll}5 & 6 & 0.1092 & 1.7165 & 10.1139 & 1.5274 & -1.0000 & -1.0000\end{array}$

$\begin{array}{llllllll}1 & 6 & 0.1485 & 1.3609 & 11.8373 & 1.3335 & 1.4000 & -1.0000\end{array}$

$\begin{array}{llllllll}6 & 7 & 0.1435 & 1.0021 & 9.7624 & 1.7440 & -1.0000 & -1.0000\end{array}$

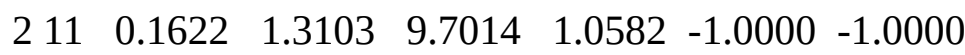

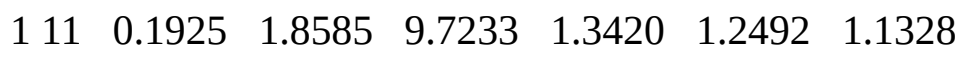

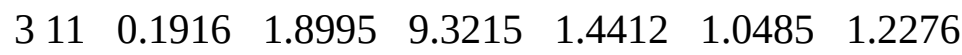

$\begin{array}{llllllll}110 & 0.0500 & 1.7500 & 12.3500 & 0.1000 & -1.0000 & -1.0000\end{array}$ 


$$
\begin{array}{cccccccc}
210 & 0.0300 & 1.5200 & 12.5000 & 0.1000 & -1.0000 & -1.0000 \\
310 & 0.0348 & 1.7637 & 12.3562 & 1.7228 & -1.0000 & -1.0000 \\
410 & 8.9905 & 1.0046 & 40.9082 & 1.8159 & -1.0226 & -1.5487 \\
11 & 10 & 0.0478 & 1.7704 & 12.8051 & 1.6100 & -1.0000 & -1.0000 \\
2 & 12 & 0.0764 & 1.5838 & 10.1462 & 1.4206 & -1.0000 & -1.0000 \\
3 & 12 & 0.1022 & 1.9887 & 10.0605 & 1.5799 & 1.4000 & -1.0000 \\
114 & \text { ! Nr of angles;at1;at2;at3;Thetao,o;ka;kb;pv1;pv2 }
\end{array}
$$

$\begin{array}{llllllllll}1 & 1 & 1 & 59.0573 & 30.7029 & 0.7606 & 0.0000 & 0.7180 & 6.2933 & 1.1244\end{array}$

$\begin{array}{llllllllll}1 & 1 & 2 & 65.7758 & 14.5234 & 6.2481 & 0.0000 & 0.5665 & 0.0000 & 1.6255\end{array}$

$\begin{array}{llllllllll}2 & 1 & 2 & 70.2607 & 25.2202 & 3.7312 & 0.0000 & 0.0050 & 0.0000 & 2.7500\end{array}$

$\begin{array}{llllllllll}1 & 2 & 2 & 0.0000 & 0.0000 & 6.0000 & 0.0000 & 0.0000 & 0.0000 & 1.0400\end{array}$

$\begin{array}{llllllllll}1 & 2 & 1 & 0.0000 & 3.4110 & 7.7350 & 0.0000 & 0.0000 & 0.0000 & 1.0400\end{array}$

$\begin{array}{llllllllll}2 & 2 & 2 & 0.0000 & 27.9213 & 5.8635 & 0.0000 & 0.0000 & 0.0000 & 1.0400\end{array}$

$\begin{array}{llllllllll}1 & 1 & 3 & 54.7427 & 21.1992 & 1.0613 & 0.0000 & 2.9950 & 58.6562 & 1.1232\end{array}$

$\begin{array}{llllllllll}3 & 1 & 3 & 78.6632 & 16.3065 & 6.3613 & -19.9300 & 1.5183 & 0.0000 & 2.2234\end{array}$

$\begin{array}{llllllllll}2 & 1 & 3 & 50.0000 & 12.9103 & 2.5311 & 0.0000 & 0.1000 & 0.0000 & 1.0000\end{array}$

$\begin{array}{llllllllll}1 & 3 & 1 & 71.6401 & 45.0000 & 1.2667 & 0.0000 & 2.8294 & 0.0000 & 1.0000\end{array}$

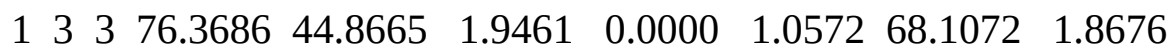

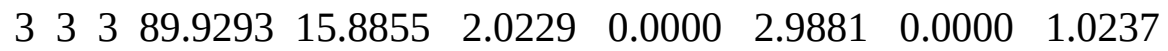

$\begin{array}{llllllllll}1 & 3 & 2 & 90.0000 & 6.6459 & 5.2255 & 0.0000 & 1.3111 & 0.0000 & 3.0000\end{array}$

$\begin{array}{llllllllll}2 & 3 & 3 & 75.6935 & 50.0000 & 2.0000 & 0.0000 & 1.0000 & 0.0000 & 1.1680\end{array}$

$\begin{array}{llllllllll}2 & 3 & 2 & 85.8000 & 9.8453 & 2.2720 & 0.0000 & 2.8635 & 0.0000 & 1.5800\end{array}$

$\begin{array}{llllllllll}1 & 2 & 3 & 0.0000 & 16.7302 & 1.1143 & 0.0000 & 0.0000 & 0.0000 & 1.0000\end{array}$

$\begin{array}{llllllllll}3 & 2 & 3 & 0.0000 & 15.0000 & 2.8900 & 0.0000 & 0.0000 & 0.0000 & 2.8774\end{array}$

$\begin{array}{llllllllll}2 & 2 & 3 & 0.0000 & 8.5744 & 3.0000 & 0.0000 & 0.0000 & 0.0000 & 1.0421\end{array}$

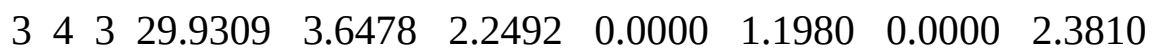

$\begin{array}{llllllllll}4 & 3 & 4 & 3.3622 & 10.1592 & 2.3865 & 0.0000 & 3.2489 & 0.0000 & 2.6812\end{array}$

$\begin{array}{llllllllll}3 & 3 & 4 & 11.7872 & 50.0000 & 5.8383 & 0.0000 & 4.6977 & 0.0000 & 1.0000\end{array}$

$\begin{array}{llllllllll}3 & 4 & 4 & 15.3871 & 4.8803 & 8.3929 & 0.0000 & 4.1095 & 0.0000 & 3.4321\end{array}$

$\begin{array}{llllllllll}1 & 3 & 4 & 90.0000 & 11.8185 & 3.0517 & 0.0000 & 2.1225 & 0.0000 & 1.0000\end{array}$

$\begin{array}{llllllllll}2 & 5 & 2 & 25.9881 & 0.0100 & 1.8827 & 0.0000 & 0.6581 & 0.0000 & 1.1500\end{array}$

$\begin{array}{llllllllll}5 & 2 & 5 & 0.0000 & 3.6249 & 1.0000 & 0.0000 & 1.0000 & 0.0000 & 1.2500\end{array}$

$\begin{array}{llllllllll}3 & 5 & 3 & 10.0000 & 0.1000 & 1.0302 & 0.0000 & 1.0000 & 0.0000 & 1.0000\end{array}$

$\begin{array}{llllllllll}3 & 3 & 5 & 106.0264 & 7.0152 & 0.2086 & 0.0000 & 0.0100 & 0.0000 & 2.5367\end{array}$

$\begin{array}{llllllllll}1 & 3 & 5 & 92.0242 & 0.1000 & 10.0000 & 0.0000 & 2.8844 & 0.0000 & 1.1706\end{array}$

$\begin{array}{llllllllll}3 & 1 & 5 & 100.2536 & 0.0100 & 4.2329 & 0.0000 & 1.7872 & 0.0000 & 2.3488\end{array}$

$\begin{array}{llllllllll}5 & 3 & 5 & 70.3878 & 3.0984 & 2.8121 & 0.0000 & 0.9139 & 0.0000 & 1.9378\end{array}$

$\begin{array}{llllllllll}3 & 2 & 6 & 0.0000 & 1.8088 & 0.0100 & 0.0000 & 0.0000 & 0.0000 & 1.2229\end{array}$

$\begin{array}{llllllllll}2 & 6 & 2 & 0.0000 & 5.6384 & 1.3636 & 0.0000 & 0.4652 & 0.0000 & 1.0400\end{array}$

$\begin{array}{llllllllll}6 & 4 & 6 & 19.0109 & 16.8964 & 13.2508 & 0.0000 & -0.3146 & 0.0000 & 1.3785\end{array}$

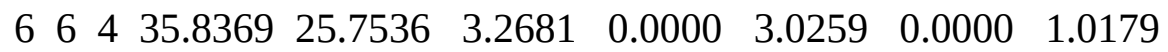

$\begin{array}{llllllllll}3 & 4 & 6 & 23.3113 & 15.3421 & 1.7515 & 0.0000 & 2.0263 & 0.0000 & 1.3581\end{array}$

$\begin{array}{llllllllll}5 & 4 & 3 & 59.8446 & 13.2857 & 0.3263 & 0.0000 & 4.0719 & 0.0000 & 1.1745\end{array}$

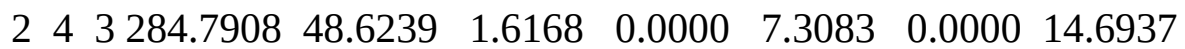

$\begin{array}{llllllllll}4 & 3 & 2 & 48.8774 & 12.4454 & 1.0604 & 0.0000 & 4.1897 & 0.0000 & 1.0077\end{array}$

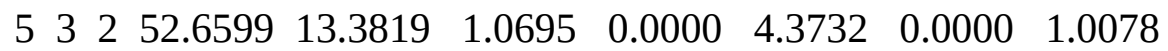

$\begin{array}{llllllllll}6 & 2 & 6 & 10.0000 & 7.7335 & 1.0000 & 0.0000 & 1.2806 & 0.0000 & 1.0400\end{array}$

$\begin{array}{llllllllll}2 & 6 & 6 & 10.0000 & 10.0000 & 3.8265 & 0.0000 & 1.0000 & 0.0000 & 1.0400\end{array}$

$\begin{array}{llllllllll}6 & 6 & 6 & 59.0573 & 30.7029 & 0.7606 & 0.0000 & 0.7180 & 6.2933 & 1.1244\end{array}$

$\begin{array}{llllllllll}2 & 2 & 6 & 10.0000 & 7.2139 & 3.7598 & 0.0000 & 1.0000 & 0.0000 & 1.7496\end{array}$

$\begin{array}{llllllllll}6 & 1 & 6 & 66.7242 & 16.7256 & 8.4462 & 0.0000 & 1.0455 & 0.0000 & 3.9255\end{array}$

$\begin{array}{llllllllll}6 & 1 & 2 & 70.0840 & 25.3540 & 3.4508 & 0.0000 & 1.0000 & 0.0000 & 3.0000\end{array}$ 


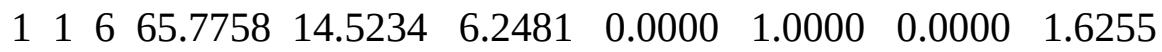

$\begin{array}{llllllllll}1 & 6 & 6 & 10.0000 & 2.3487 & 6.0000 & 0.0000 & 5.0000 & 0.0000 & 1.0000\end{array}$

$\begin{array}{llllllllll}1 & 6 & 1 & 10.0000 & 3.4110 & 7.7350 & 0.0000 & 1.0000 & 0.0000 & 1.0400\end{array}$

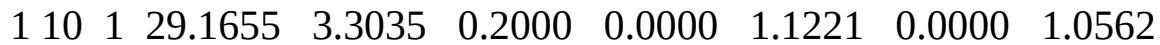

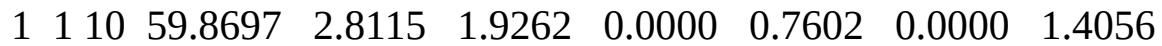

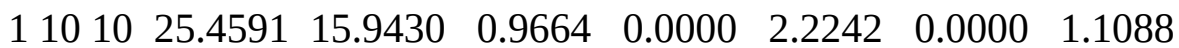

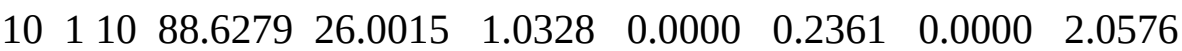

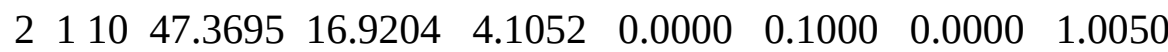

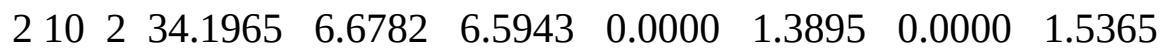

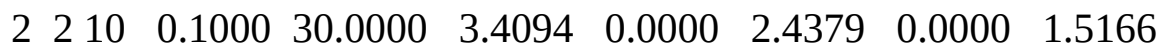

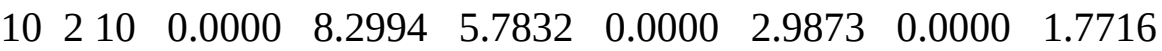

$\begin{array}{lllllllll}210 & 10 & 21.2590 & 6.5954 & 0.9951 & 0.0000 & 2.8006 & 0.0000 & 1.0000\end{array}$

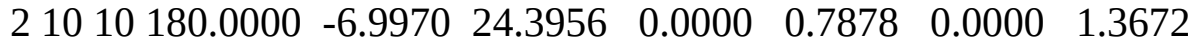

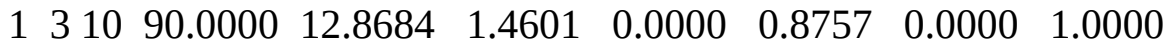

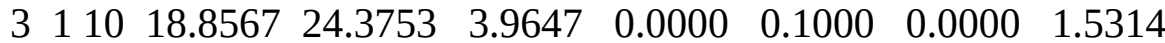

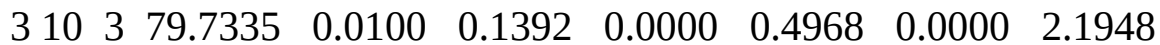

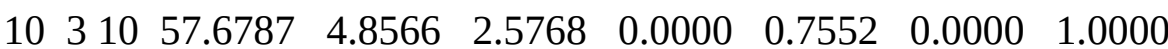

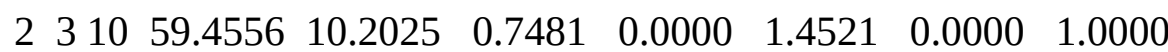

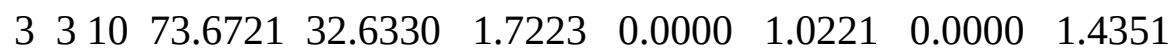

$\begin{array}{lllllllll}310 & 10 & 65.7545 & 5.6268 & 4.0645 & 0.0000 & 1.7794 & 0.0000 & 2.6730\end{array}$

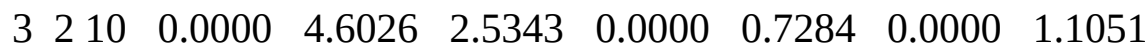

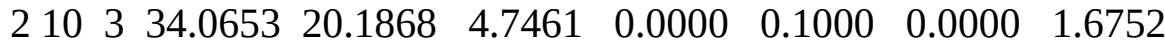

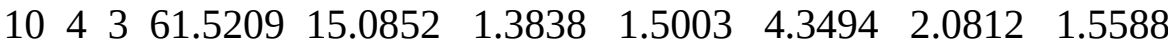

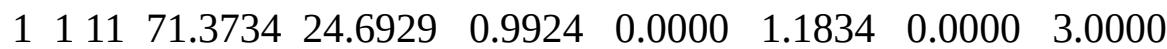

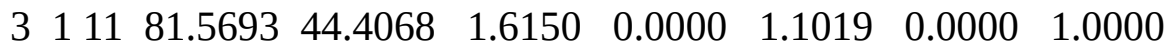

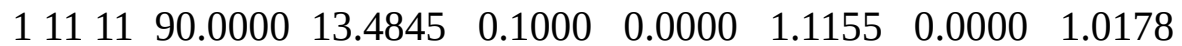

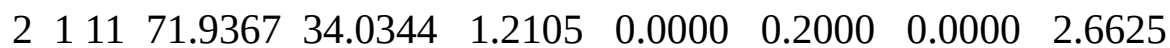

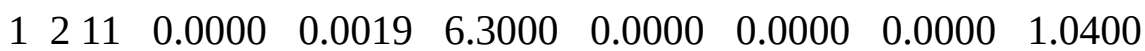

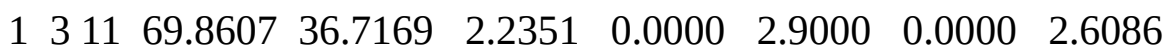

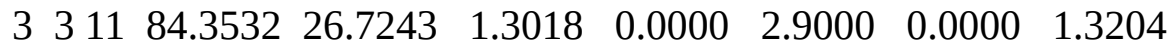

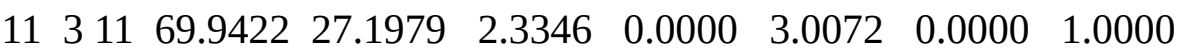

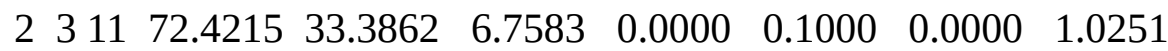

$\begin{array}{lllllllll}111 & 1 & 78.5107 & 22.7928 & 1.2062 & 0.0000 & 2.8702 & 0.0000 & 1.0000\end{array}$

$\begin{array}{lllllllll}111 & 3 & 74.7913 & 29.2724 & 1.1363 & 0.0000 & 2.8701 & 0.0000 & 1.5851\end{array}$

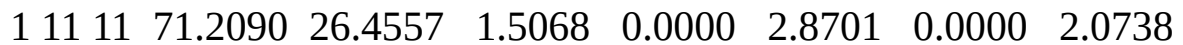

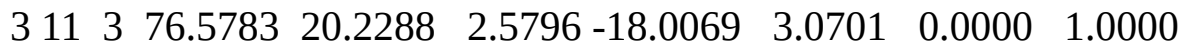

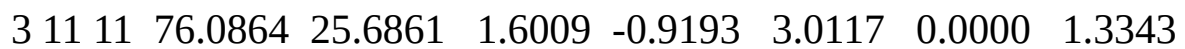

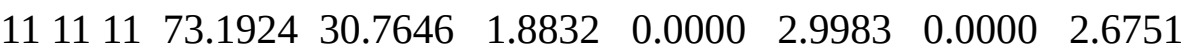

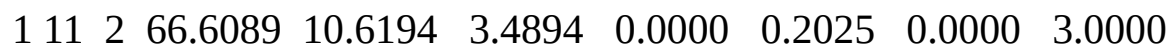

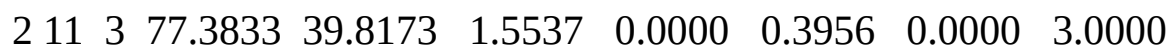

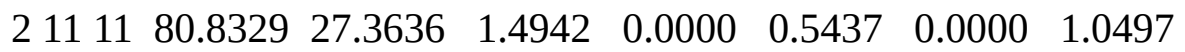

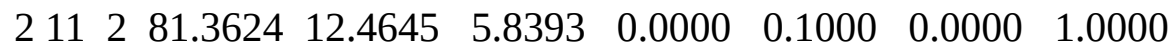

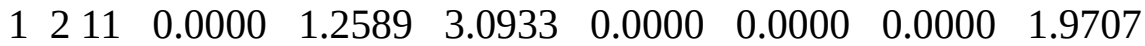

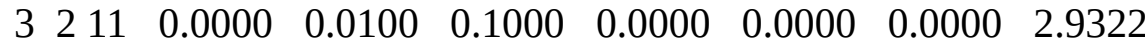

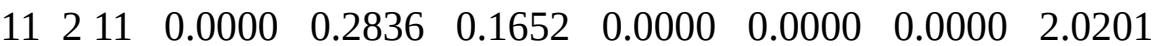

$\begin{array}{llllllllll}2 & 2 & 3 & 0.0000 & 8.5744 & 3.0000 & 0.0000 & 0.0000 & 0.0000 & 1.0421\end{array}$

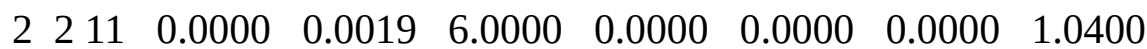

$\begin{array}{lllllllll}310 & 3 & 96.2265 & 4.5610 & 12.0000 & 0.0000 & 0.3211 & 0.0000 & 1.5204\end{array}$

$\begin{array}{lllllllll}310 & 3 & 0.0000 & 9.1552 & 7.9919 & 0.0000 & 0.1660 & 0.0000 & 1.5386\end{array}$

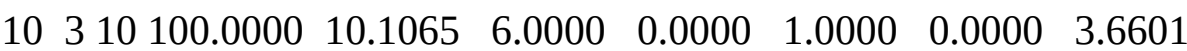

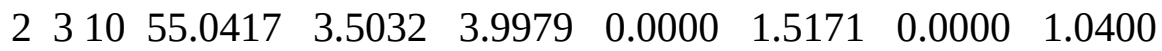

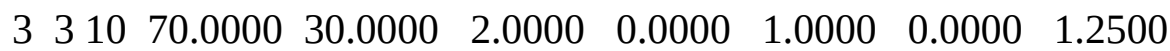




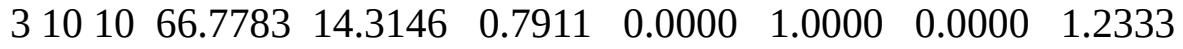

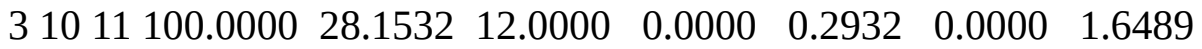

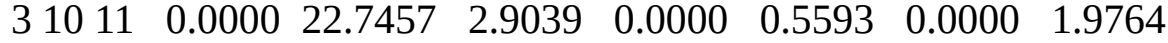

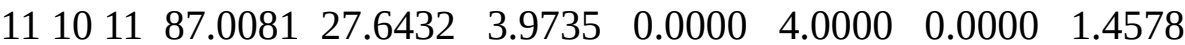

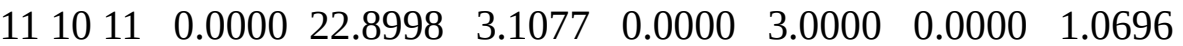

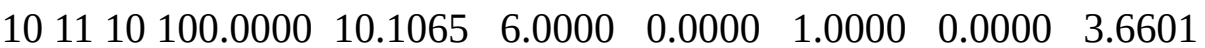

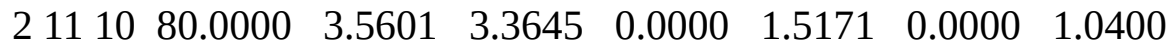

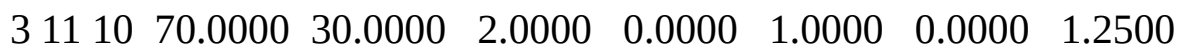

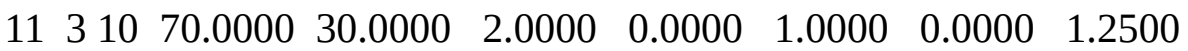

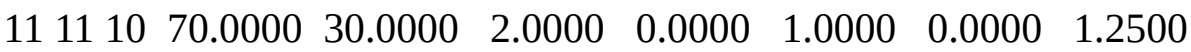

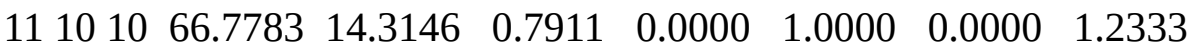

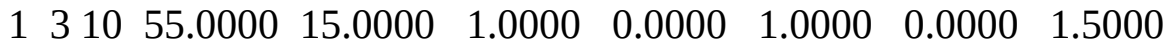

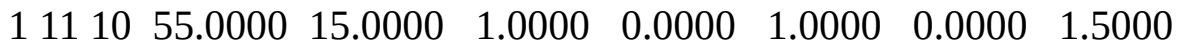

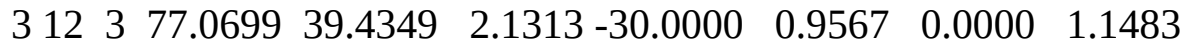

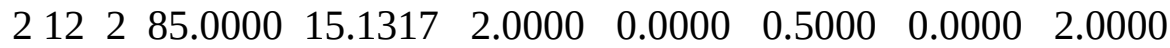

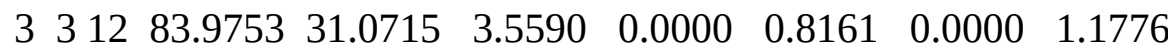

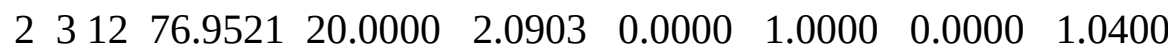
53 ! Nr of torsions;at1;at2;at3;at4;;V1;V2;V3;V2(BO);vconj;n.u;n

$\begin{array}{lllllllllll}1 & 1 & 1 & 1 & -0.2500 & 34.7453 & 0.0288 & -6.3507 & -1.6000 & 0.0000 & 0.0000\end{array}$

$\begin{array}{lllllllllll}1 & 1 & 1 & 2 & -0.2500 & 29.2131 & 0.2945 & -4.9581 & -2.1802 & 0.0000 & 0.0000\end{array}$

$\begin{array}{lllllllllll}2 & 1 & 1 & 2 & -0.2500 & 31.2081 & 0.4539 & -4.8923 & -2.2677 & 0.0000 & 0.0000\end{array}$

$\begin{array}{llllllllllll}1 & 1 & 1 & 3 & -2.5000 & 25.4016 & 1.0000 & -4.4850 & -1.1000 & 0.0000 & 0.0000\end{array}$

$\begin{array}{lllllllllll}2 & 1 & 1 & 3 & -0.9763 & 59.4161 & 1.0000 & -7.7414 & -1.0978 & 0.0000 & 0.0000\end{array}$

$\begin{array}{lllllllllll}3 & 1 & 1 & 3 & -2.5000 & 52.7614 & -1.0000 & -4.0134 & -0.8614 & 0.0000 & 0.0000\end{array}$

$\begin{array}{lllllllllll}1 & 1 & 3 & 1 & -1.9125 & 80.0000 & -1.0000 & -4.5626 & -0.9000 & 0.0000 & 0.0000\end{array}$

$\begin{array}{lllllllllll}1 & 1 & 3 & 2 & 0.6154 & 8.3019 & -0.4870 & -2.9336 & -0.9000 & 0.0000 & 0.0000\end{array}$

$\begin{array}{llllllllllll}2 & 1 & 3 & 1 & -2.5000 & 80.0000 & 0.9658 & -4.4935 & -0.9000 & 0.0000 & 0.0000\end{array}$

$\begin{array}{lllllllllll}2 & 1 & 3 & 2 & -1.0000 & 31.8695 & 1.0000 & -2.6151 & -1.1000 & 0.0000 & 0.0000\end{array}$

$\begin{array}{lllllllllll}1 & 1 & 3 & 3 & 0.7514 & 34.1941 & 0.5669 & -5.5360 & -2.0544 & 0.0000 & 0.0000\end{array}$

$\begin{array}{lllllllllll}2 & 1 & 3 & 3 & 2.5000 & 80.0000 & 1.0000 & -2.6841 & -2.8274 & 0.0000 & 0.0000\end{array}$

$\begin{array}{lllllllllll}3 & 1 & 3 & 1 & 0.2515 & 79.1495 & -0.6263 & -4.3647 & -3.0437 & 0.0000 & 0.0000\end{array}$

$\begin{array}{lllllllllll}3 & 1 & 3 & 2 & 1.0000 & 37.1243 & 1.0000 & -2.5000 & -3.0476 & 0.0000 & 0.0000\end{array}$

$\begin{array}{lllllllllll}3 & 1 & 3 & 3 & -1.0092 & 41.0504 & 0.3915 & -6.0913 & -2.7174 & 0.0000 & 0.0000\end{array}$

$\begin{array}{lllllllllll}1 & 3 & 3 & 1 & -1.6378 & -11.8357 & 0.3815 & -3.2104 & -2.7536 & 0.0000 & 0.0000\end{array}$

$\begin{array}{lllllllllll}1 & 3 & 3 & 2 & -2.5000 & -9.2805 & 0.3063 & -5.9187 & -2.9498 & 0.0000 & 0.0000\end{array}$

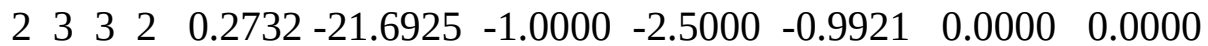

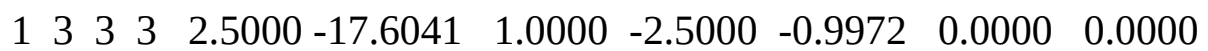

$\begin{array}{lllllllllll}2 & 3 & 3 & 3 & -2.5000 & 78.0855 & -0.8750 & -7.8902 & -1.2407 & 0.0000 & 0.0000\end{array}$

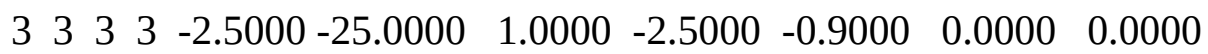

$\begin{array}{lllllllllll}0 & 1 & 2 & 0 & 0.0000 & 0.0000 & 0.0000 & 0.0000 & 0.0000 & 0.0000 & 0.0000\end{array}$

$\begin{array}{llllllllllll}0 & 2 & 2 & 0 & 0.0000 & 0.0000 & 0.0000 & 0.0000 & 0.0000 & 0.0000 & 0.0000\end{array}$

$\begin{array}{lllllllllll}0 & 2 & 3 & 0 & 0.0000 & 0.1000 & 0.0200 & -2.5415 & 0.0000 & 0.0000 & 0.0000\end{array}$

$\begin{array}{llllllllllll}0 & 1 & 1 & 0 & 0.0000 & 50.0000 & 0.3000 & -4.0000 & -2.0000 & 0.0000 & 0.0000\end{array}$

$\begin{array}{lllllllllll}0 & 3 & 3 & 0 & 0.5511 & 25.4150 & 1.1330 & -5.1903 & -1.0000 & 0.0000 & 0.0000\end{array}$

$\begin{array}{lllllllllll}0 & 1 & 6 & 0 & 3.3423 & 30.3435 & 0.0365 & -2.7171 & 0.0000 & 0.0000 & 0.0000\end{array}$

$\begin{array}{lllllllllll}0 & 6 & 6 & 0 & -0.0500 & 10.0000 & 0.1565 & -2.2006 & 0.0000 & 0.0000 & 0.0000\end{array}$ $\begin{array}{lllllllllll}0 & 2 & 6 & 0 & 0.0000 & 0.0000 & 0.0000 & 0.0000 & 0.0000 & 0.0000 & 0.0000\end{array}$

$\begin{array}{lllllllllll}1 & 1 & 1 & 6 & -0.2500 & 29.2131 & 0.2945 & -4.9581 & -2.1802 & 0.0000 & 0.0000\end{array}$

$\begin{array}{lllllllllll}6 & 1 & 1 & 6 & -0.2500 & 31.2081 & 0.4539 & -4.8923 & -2.2677 & 0.0000 & 0.0000\end{array}$

$\begin{array}{lllllllllll}6 & 6 & 6 & 6 & -0.2500 & 34.7453 & 0.0288 & -6.3507 & -1.6000 & 0.0000 & 0.0000\end{array}$

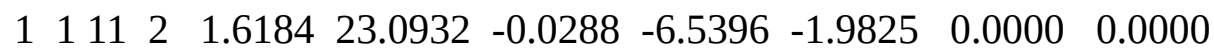

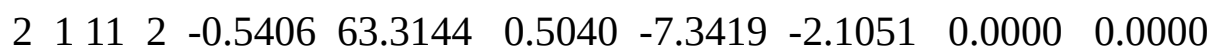




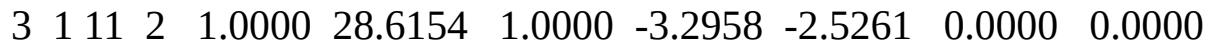

$\begin{array}{llllllllllll}3 & 1 & 1 & 11 & -0.5652 & 57.5647 & 0.8258 & -4.8580 & -0.9511 & 0.0000 & 0.0000\end{array}$

$\begin{array}{lllllllllll}11 & 1 & 1 & 11 & 0.6625 & 64.6500 & 0.1720 & -3.7433 & -1.7241 & 0.0000 & 0.0000\end{array}$

$\begin{array}{lllllllllll}1 & 1 & 11 & 1 & 1.0000 & 11.8739 & 0.7164 & -4.7719 & -1.6589 & 0.0000 & 0.0000\end{array}$

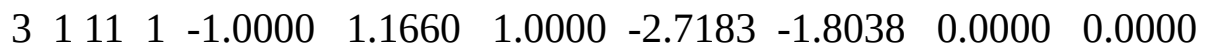

$\begin{array}{lllllllllll}2 & 1 & 1 & 11 & 1.0000 & 27.4158 & 0.3693 & -3.5264 & -1.9000 & 0.0000 & 0.0000\end{array}$

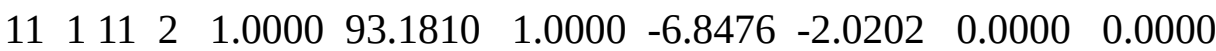

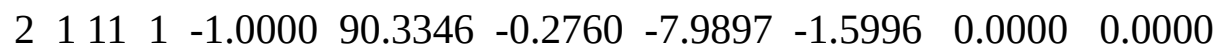

$\begin{array}{lllllllllll}0 & 1 & 11 & 0 & 0.2176 & 40.4126 & 0.3535 & -3.9875 & -2.0051 & 0.0000 & 0.0000\end{array}$

$\begin{array}{lllllllllll}0 & 2 & 11 & 0 & 0.0000 & 0.1032 & 0.3000 & -5.0965 & 0.0000 & 0.0000 & 0.0000\end{array}$

$\begin{array}{lllllllllll}0 & 3 & 11 & 0 & 1.1397 & 61.3225 & 0.5139 & -3.8507 & -2.7831 & 0.0000 & 0.0000\end{array}$

$\begin{array}{llllllllll}011 & 11 & 0 & 0.7265 & 44.3155 & 1.0000 & -4.4046 & -2.0000 & 0.0000 & 0.0000\end{array}$

$\begin{array}{lllllllllll}11 & 1 & 11 & 11 & -0.0949 & 8.7582 & 0.3310 & -7.9430 & -2.0000 & 0.0000 & 0.0000\end{array}$

$\begin{array}{lllllllllll}2 & 3 & 10 & 3 & -1.5000 & 6.8333 & -0.1978 & -1.4683 & 0.0000 & 0.0000 & 0.0000\end{array}$

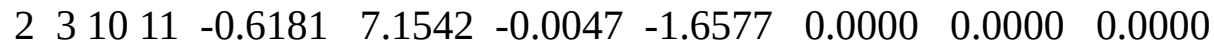

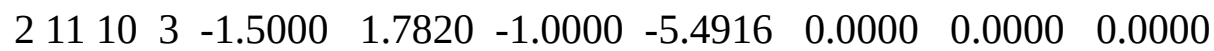

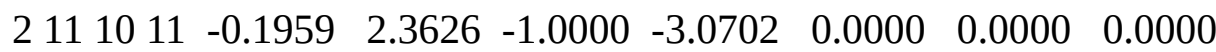

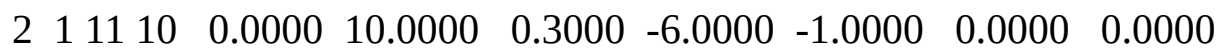

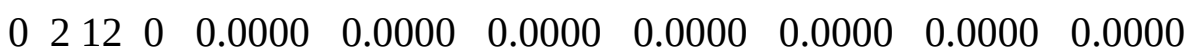

4 ! Nr of hydrogen bonds;at1;at2;at3;Rhb;Dehb;vhb1

$\begin{array}{lllllll}3 & 2 & 3 & 0.7000 & -3.5800 & 1.4500 & 19.5000\end{array}$

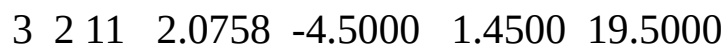

$\begin{array}{lllllll}11 & 2 & 3 & 2.1744 & -1.6397 & 1.4500 & 19.5000\end{array}$

$\begin{array}{lllllll}11 & 2 & 11 & 1.9250 & -2.4190 & 1.4500 & 19.5000\end{array}$ 


\section{$\mathrm{Fe} / \mathrm{Mn} / \mathrm{O} / \mathrm{C} / \mathrm{H}$ Force field:}

Refinement of:

Fe: c/h/o/water/Fe Aryanpour et al JPC-A 2010 ReaxFF(full)

Mn: Electrode-electrolyte interface layers in lithium ion batteries using reactive force field based molecular dynamics by Sahithya Reddivari, doctoral thesis, 2016, University of Michigan

Reactive MD-force field: Mn/Fe/O/C/H Gomzi et al 2021.

39 ! Number of general parameters

50.0000 !Overcoordination parameter

9.5469 !Overcoordination parameter

1.6725 !Valency angle conjugation parameter

1.7224 !Triple bond stabilisation parameter

6.8702 !Triple bond stabilisation parameter

60.4850 !C2-correction

1.0588 !Undercoordination parameter

4.6000 !Triple bond stabilisation parameter

12.1176 !Undercoordination parameter

13.3056 !Undercoordination parameter

-55.1978 !Triple bond stabilization energy

0.0000 !Lower Taper-radius

10.0000 !Upper Taper-radius

2.8793 !Not used

33.8667 !Valency undercoordination

6.0891 !Valency angle/lone pair parameter

1.0563 !Valency angle

2.0384 !Valency angle parameter

6.1431 !Not used

6.9290 !Double bond/angle parameter

0.3989 !Double bond/angle parameter: overcoord

3.9954 !Double bond/angle parameter: overcoord

-2.4837 !Not used

5.7796 !Torsion/BO parameter

10.0000 !Torsion overcoordination

1.9487 !Torsion overcoordination

-1.2327 !Conjugation 0 (not used)

2.1645 !Conjugation

1.5591 !vdWaals shielding

0.1000 !Cutoff for bond order $(* 100)$

1.7602 !Valency angle conjugation parameter

0.6991 !Overcoordination parameter

50.0000 !Overcoordination parameter

1.8512 !Valency/lone pair parameter

0.5000 !Not used

20.0000 !Not used

5.0000 !Molecular energy (not used)

0.0000 !Molecular energy (not used)

0.7903 !Valency angle conjugation parameter

10 ! Nr of atoms; cov.r; valency;a.m;Rvdw;Evdw;gammaEEM;cov.r2;

alfa;gammavdW;valency;Eunder;Eover;chiEEM;etaEEM;n.u.

cov r3;Elp;Heat inc.;n.u.;n.u.;n.u.;n.u.

ov/un;val1;n.u.;val3,vval4 
$\begin{array}{lllllllll}\text { C } & 1.3817 & 4.0000 & 12.0000 & 1.8903 & 0.1838 & 0.6387 & 1.1341 & 4.0000\end{array}$ $\begin{array}{llllllll}9.7559 & 2.1346 & 4.0000 & 34.9350 & 79.5548 & 4.9218 & 6.0000 & 0.0000\end{array}$

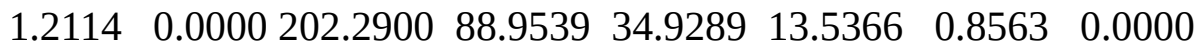
$\begin{array}{llllllll}-2.8983 & 2.5000 & 1.0560 & 44.0000 & 2.9663 & 0.0000 & 0.0000 & 0.0000\end{array}$

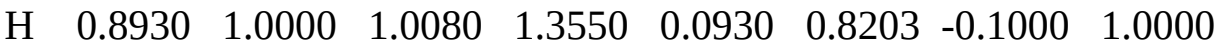
$\begin{array}{llllllll}8.2230 & 33.2894 & 1.0000 & 0.0000 & 121.1250 & 3.7248 & 9.6093 & 1.0000\end{array}$ $\begin{array}{llllllll}-0.1000 & 0.0000 & 55.1870 & 83.0408 & 2.4197 & 0.0003 & 1.0698 & 0.0000\end{array}$ $\begin{array}{llllllll}-19.4571 & 4.2733 & 1.0330 & 81.0000 & 2.8793 & 0.0000 & 0.0000 & 0.0000\end{array}$

$\begin{array}{lllllllll}\text { O } & 1.2450 & 2.0000 & 15.9990 & 2.3890 & 0.1000 & 1.0898 & 1.0548 & 6.0000\end{array}$ $\begin{array}{llllllll}9.7300 & 13.8449 & 4.0000 & 37.5000 & 116.0768 & 8.5000 & 8.3122 & 2.0000\end{array}$ $\begin{array}{llllllll}0.9049 & 0.4056 & 68.0152 & 3.5027 & 0.7640 & 0.0021 & 0.9745 & 0.0000\end{array}$ $\begin{array}{llllllll}-3.5500 & 2.9000 & 1.0493 & 4.0000 & 2.9225 & 0.0000 & 0.0000 & 0.0000\end{array}$

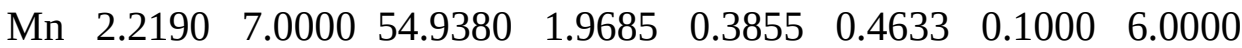
$\begin{array}{llllllll}11.2058 & 4.1928 & 4.0000 & 0.0000 & 0.0000 & -1.0000 & 6.1911 & 0.0000\end{array}$ $\begin{array}{llllllll}0.1000 & 0.0000 & 152.6300 & 3.4529 & 0.0722 & 3.1767 & 0.8563 & 0.0000\end{array}$ $\begin{array}{llllllll}-25.0000 & 3.1072 & 1.0338 & 8.0000 & 3.4590 & 0.0000 & 0.0000 & 0.0000\end{array}$

$\begin{array}{lllllllll}\mathrm{Li} & 1.9814 & 1.0000 & 6.9410 & 1.8000 & 0.2939 & 0.9387 & -0.1000 & 1.0000\end{array}$ $\begin{array}{llllllll}9.0616 & 1.3258 & 1.0000 & 0.0000 & 0.0000 & -3.0000 & 10.0241 & 0.0000\end{array}$ $\begin{array}{llllllll}-1.0000 & 0.0000 & 37.5000 & 5.4409 & 6.9107 & 0.1973 & 0.8563 & 0.0000\end{array}$ $\begin{array}{llllllll}-2.5068 & 2.2989 & 1.0338 & 1.0000 & 2.8103 & 1.3000 & 0.2000 & 13.0000\end{array}$

F $\quad \begin{array}{llllllll}1.7938 & 1.0000 & 18.9984 & 1.4139 & 0.3134 & 0.7750 & -0.1000 & 7.0000\end{array}$ $\begin{array}{llllllll}10.3051 & 15.1397 & 1.0000 & 9.2533 & 0.2000 & 9.8105 & 8.6941 & 0.0000\end{array}$ $\begin{array}{llllllll}-1.0000 & 3.5571 & 18.0000 & 6.9821 & 4.1799 & 1.0561 & 0.0000 & 0.0000\end{array}$ $\begin{array}{llllllll}-6.3417 & 2.6656 & 1.0493 & 4.0000 & 2.9225 & 0.0000 & 0.0000 & 0.0000\end{array}$

$\begin{array}{lllllllll}\mathrm{P} & 1.5994 & 3.0000 & 30.9738 & 1.7000 & 0.1743 & 1.0385 & 1.3000 & 5.0000\end{array}$ $\begin{array}{lllllllll}9.1909 & 14.2932 & 5.0000 & 0.0000 & 0.0000 & 0.9528 & 7.9121 & 0.0000\end{array}$ $\begin{array}{llllllll}-1.0000 & 10.2596 & 1.5000 & 0.2205 & 16.7429 & 15.9629 & 0.0000 & 0.0000\end{array}$ $\begin{array}{llllllll}-2.5000 & 1.6114 & 1.0338 & 5.0000 & 2.8793 & 0.0000 & 0.0000 & 0.0000\end{array}$

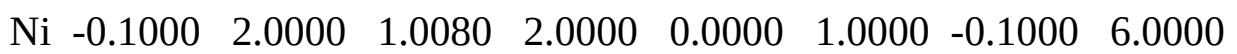
$\begin{array}{lllllllll}10.0000 & 2.5000 & 4.0000 & 0.0000 & 0.0000 & 8.5000 & 1.5000 & 0.0000\end{array}$ $\begin{array}{llllllll}-0.1000 & 0.0000 & -2.3700 & 8.7410 & 13.3640 & 0.6690 & 0.9745 & 0.0000\end{array}$ $\begin{array}{llllllll}-11.0000 & 2.7466 & 1.0338 & 6.2998 & 2.8793 & 0.0000 & 0.0000 & 0.0000\end{array}$

$\begin{array}{lllllllll}\mathrm{Al} & -0.1000 & 2.0000 & 1.0080 & 2.0000 & 0.0000 & 1.0000 & -0.1000 & 6.0000\end{array}$ $\begin{array}{llllllll}10.0000 & 2.5000 & 4.0000 & 0.0000 & 0.0000 & 8.5000 & 1.5000 & 0.0000\end{array}$ $\begin{array}{llllllll}-0.1000 & 0.0000 & -2.3700 & 8.7410 & 13.3640 & 0.6690 & 0.9745 & 0.0000\end{array}$ $\begin{array}{llllllll}-11.0000 & 2.7466 & 1.0338 & 6.2998 & 2.8793 & 0.0000 & 0.0000 & 0.0000\end{array}$

Fe $1.9506 \quad 3.0000 \quad 55.8450 \quad 2.0308 \quad 0.1274 \quad 0.7264 \quad-1.0000 \quad 3.0000$ $\begin{array}{llllllll}11.0534 & 2.2637 & 3.0000 & 0.0000 & 18.3725 & 1.2457 & 7.3021 & 0.0000\end{array}$ $\begin{array}{llllllll}-1.2000 & 0.0000 & 66.4838 & 30.0000 & 1.0000 & 0.0000 & 0.8563 & 0.0000\end{array}$ $\begin{array}{llllllll}-16.2040 & 2.7917 & 1.0338 & 6.0000 & 2.5791 & 0.0000 & 0.0000 & 0.0000\end{array}$

$33 \quad$ ! Nr of bonds; Edis1;LPpen;n.u.;pbe1;pbo5;13corr;pbo6 pbe2;pbo3;pbo4;Etrip;pbo1;pbo2;ovcorr

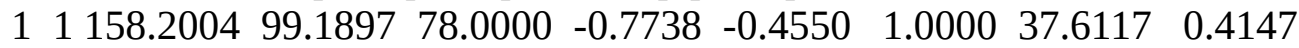
$\begin{array}{llllllll}0.4590 & -0.1000 & 9.1628 & 1.0000 & -0.0777 & 6.7268 & 1.0000 & 0.0000\end{array}$

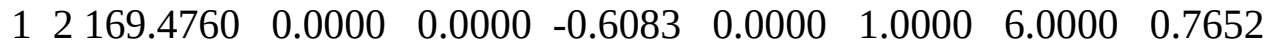
$\begin{array}{llllllll}5.2290 & 1.0000 & 0.0000 & 1.0000 & -0.0553 & 6.9316 & 0.0000 & 0.0000\end{array}$

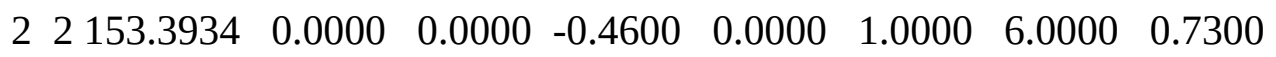
$\begin{array}{llllllll}6.2500 & 1.0000 & 0.0000 & 1.0000 & -0.0790 & 6.0552 & 0.0000 & 0.0000\end{array}$

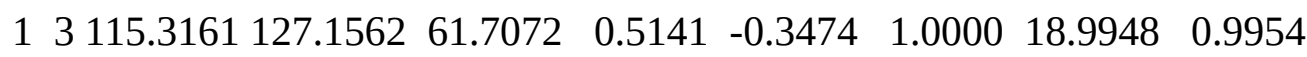
$\begin{array}{llllllll}1.5618 & -0.3414 & 8.9489 & 1.0000 & -0.1628 & 5.6821 & 0.0000 & 0.0000\end{array}$

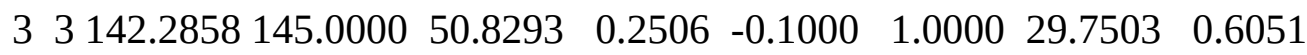
$\begin{array}{llllllll}0.3451 & -0.1055 & 9.0000 & 1.0000 & -0.1225 & 5.5000 & 1.0000 & 0.0000\end{array}$ 


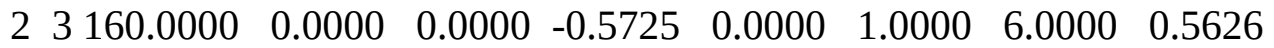
$\begin{array}{llllllll}1.1150 & 1.0000 & 0.0000 & 0.0000 & -0.0920 & 4.2790 & 0.0000 & 0.0000\end{array}$

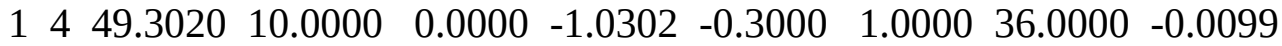
$\begin{array}{llllllll}1.8910 & -0.4204 & 7.6871 & 1.0000 & -0.0880 & 6.6676 & 1.0000 & 0.0000\end{array}$

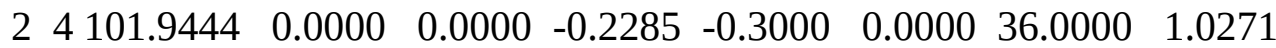
$\begin{array}{llllllll}7.0832 & -0.1532 & 19.8594 & 1.0000 & -0.0836 & 4.7210 & 0.0000 & 0.0000\end{array}$

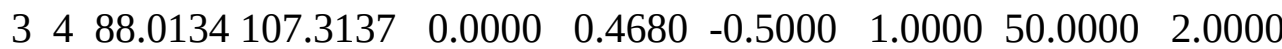
$\begin{array}{llllllll}7.5815 & -0.4509 & 7.6953 & 1.0000 & -0.0952 & 5.1424 & 1.0000 & 0.0000\end{array}$

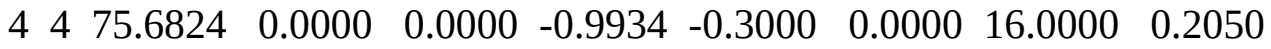
$\begin{array}{llllllll}2.2857 & -0.3000 & 16.0000 & 1.0000 & -0.1230 & 8.7005 & 0.0000 & 0.0000\end{array}$

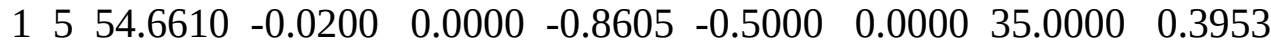
$\begin{array}{llllllll}0.6908 & -0.2500 & 11.9965 & 1.0000 & -0.0668 & 9.0596 & 0.0000 & 0.0000\end{array}$

$\begin{array}{llllllllll}2 & 5 & 59.2034 & 0.0000 & 0.0000 & 0.1240 & 0.0000 & 0.0000 & 6.0000 & 0.4000\end{array}$ $\begin{array}{llllllll}1.0000 & 0.0000 & 12.0000 & 1.0000 & -0.0565 & 4.9575 & 0.0000 & 0.0000\end{array}$

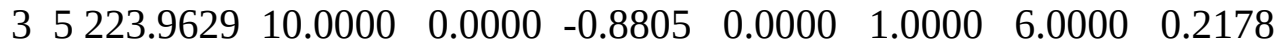
$\begin{array}{llllllll}4.2326 & -0.1048 & 7.0167 & 1.0000 & -0.0867 & 4.2825 & 0.0000 & 0.0000\end{array}$

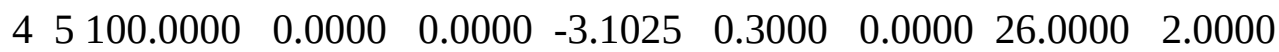
$\begin{array}{llllllll}3.2823 & 0.0000 & 12.0000 & 1.0000 & -0.0800 & 6.9752 & 0.0000 & 0.0000\end{array}$

$\begin{array}{llllllllll}5 & 5 & 34.3154 & 0.0000 & 0.0000 & 0.5995 & 0.3000 & 0.0000 & 26.0000 & 0.5445\end{array}$ $\begin{array}{llllllll}0.5752 & 0.0000 & 12.0000 & 1.0000 & -0.1382 & 4.5000 & 0.0000 & 0.0000\end{array}$

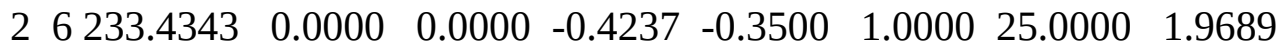
$\begin{array}{llllllll}4.7870 & -0.2500 & 15.0000 & 1.0000 & -0.1288 & 4.9159 & 1.0000 & 0.0000\end{array}$

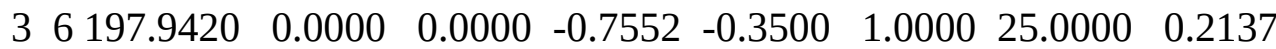
$\begin{array}{llllllll}9.5888 & -0.2500 & 15.0000 & 1.0000 & -0.1477 & 7.8947 & 1.0000 & 0.0000\end{array}$

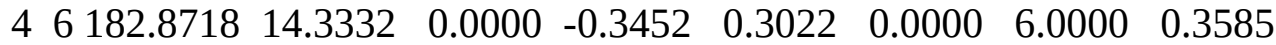
$\begin{array}{llllllll}2.6684 & -0.1484 & 11.8165 & 1.0000 & -0.0940 & 5.8491 & 0.0000 & 0.0000\end{array}$

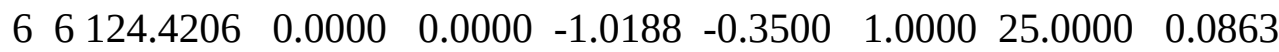
$\begin{array}{llllllll}8.4257 & -0.2500 & 15.0000 & 1.0000 & -0.2337 & 8.1452 & 1.0000 & 0.0000\end{array}$

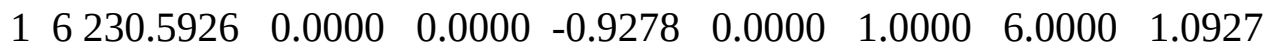
$\begin{array}{llllllll}7.1644 & 1.0000 & 0.0000 & 1.0000 & -0.0732 & 5.9745 & 0.0000 & 0.0000\end{array}$

$\begin{array}{llllllllll}5 & 6 & 92.6032 & 0.0000 & 0.0000 & -0.5454 & -0.5582 & 0.0000 & 44.9378 & 0.3238\end{array}$ $\begin{array}{llllllll}1.0563 & -0.2500 & 15.0000 & 1.0000 & -0.1206 & 4.0000 & 0.0000 & 0.0000\end{array}$

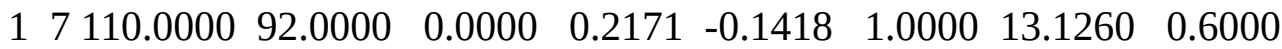
$\begin{array}{llllllll}0.3601 & -0.1310 & 10.7257 & 1.0000 & -0.0869 & 5.3302 & 1.0000 & 0.0000\end{array}$

$\begin{array}{llllllllll}2 & 7 & 0.1466 & 0.0000 & 0.0000 & 0.2250 & -0.1418 & 1.0000 & 13.1260 & 0.6000\end{array}$ $\begin{array}{llllllll}0.3912 & -0.1310 & 0.0000 & 1.0000 & -0.1029 & 9.3302 & 0.0000 & 0.0000\end{array}$

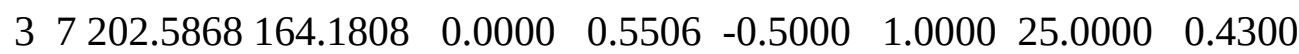
$\begin{array}{llllllll}0.0912 & -0.1285 & 16.0342 & 1.0000 & -0.2008 & 6.2678 & 1.0000 & 0.0000\end{array}$

$\begin{array}{llllllllll}7 & 7 & 0.0000 & 0.0000 & 0.0000 & 0.2171 & -0.5000 & 1.0000 & 35.0000 & 0.6000\end{array}$ $\begin{array}{llllllll}0.5000 & -0.5000 & 20.0000 & 1.0000 & -0.2000 & 10.0000 & 1.0000 & 0.0000\end{array}$

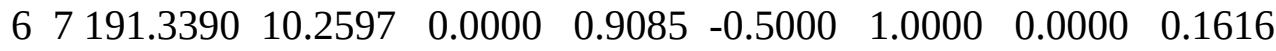
$\begin{array}{llllllll}0.0940 & -0.1039 & 10.0002 & 1.0000 & -0.1073 & 9.9960 & 1.0000 & 0.0000\end{array}$

$\begin{array}{llllllllll}5 & 7 & 0.0000 & 0.0000 & 0.0000 & 0.5000 & -0.2000 & 0.0000 & 16.0000 & 0.5000\end{array}$ $\begin{array}{llllllll}1.0001 & -0.2000 & 15.0000 & 1.0000 & -0.1000 & 10.0000 & 0.0000 & 0.0000\end{array}$

$\begin{array}{llllllllll}4 & 7 & 0.0000 & 0.0000 & 0.0000 & 0.5000 & -0.2000 & 0.0000 & 16.0000 & 0.5000\end{array}$ $\begin{array}{llllllll}1.0001 & -0.2000 & 15.0000 & 1.0000 & -0.1000 & 10.0000 & 0.0000 & 0.0000\end{array}$

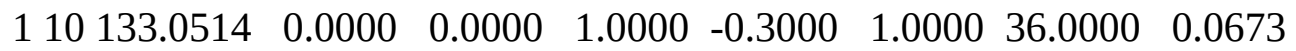
$\begin{array}{llllllll}0.2350 & -0.3500 & 15.0000 & 1.0000 & -0.1143 & 4.5217 & 1.0000 & 0.0000\end{array}$

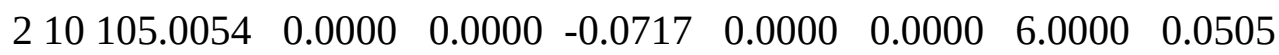
$\begin{array}{llllllll}0.1000 & 1.0000 & 0.0000 & 1.0000 & -0.1216 & 4.5062 & 0.0000 & 0.0000\end{array}$

$\begin{array}{lllllllll}310 & 65.7713 & 0.0000 & 0.0000 & 0.1366 & -0.3000 & 1.0000 & 36.0000 & 0.0494\end{array}$ $\begin{array}{llllllll}0.9495 & -0.3500 & 15.0000 & 1.0000 & -0.0555 & 7.9897 & 1.0000 & 0.0000\end{array}$ 


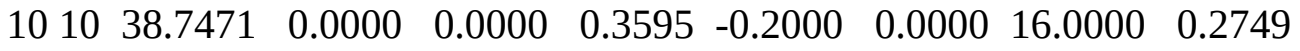
$\begin{array}{llllllll}1.0000 & -0.2000 & 15.0000 & 1.0000 & -0.0771 & 6.4477 & 0.0000 & 0.0000\end{array}$

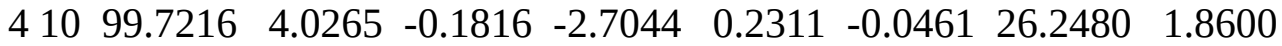
$\begin{array}{llllllll}4.1052 & 0.3126 & 12.5781 & 0.9850 & -0.0903 & 7.5452 & -0.8575 & -0.0020\end{array}$

20 ! Nr of off-diagonal terms; Ediss;Ro;gamma;rsigma;rpi;rpi2

$\begin{array}{llllllll}1 & 2 & 0.1239 & 1.4004 & 9.8467 & 1.1210 & -1.0000 & -1.0000\end{array}$

$\begin{array}{llllllll}2 & 3 & 0.0283 & 1.2885 & 10.9190 & 0.9215 & -1.0000 & -1.0000\end{array}$

$\begin{array}{llllllll}1 & 3 & 0.0503 & 1.8006 & 10.2114 & 1.3492 & 1.1992 & 1.0506\end{array}$

$\begin{array}{llllllll}1 & 4 & 0.0475 & 1.6810 & 8.8902 & 1.5153 & 1.0000 & -1.0000\end{array}$

$\begin{array}{llllllll}2 & 4 & 0.1017 & 1.3259 & 12.5406 & 1.4534 & -1.0000 & -1.0000\end{array}$

$\begin{array}{llllllll}3 & 4 & 0.1121 & 2.0879 & 11.2110 & 1.5063 & 1.4795 & -1.0000\end{array}$

$\begin{array}{llllllll}1 & 5 & 0.0270 & 2.4124 & 11.4640 & 1.7840 & 1.0000 & 1.0000\end{array}$

$\begin{array}{llllllll}2 & 5 & 0.1149 & 1.4658 & 11.0886 & 1.3337 & -1.0000 & -1.0000\end{array}$

$\begin{array}{llllllll}3 & 5 & 0.0687 & 1.8765 & 12.0451 & 1.2928 & -1.0000 & -1.0000\end{array}$

$\begin{array}{llllllll}4 & 5 & 7.1011 & 0.9961 & 40.6852 & 1.7459 & -1.0000 & -1.0000\end{array}$

$\begin{array}{llllllll}2 & 6 & 0.1087 & 1.8734 & 9.1166 & 1.0000 & -1.0000 & -1.0000\end{array}$

$\begin{array}{llllllll}3 & 6 & 0.1714 & 1.6576 & 10.7298 & 1.5553 & -1.0000 & -1.0000\end{array}$

$\begin{array}{llllllll}4 & 6 & 0.0837 & 1.9512 & 12.2762 & 1.4795 & -1.0000 & -1.0000\end{array}$

$\begin{array}{llllllll}5 & 6 & 0.1092 & 1.7165 & 10.1139 & 1.5274 & -1.0000 & -1.0000\end{array}$

$\begin{array}{llllllll}1 & 6 & 0.1485 & 1.3609 & 11.8373 & 1.3335 & 1.4000 & -1.0000\end{array}$

$\begin{array}{llllllll}6 & 7 & 0.1435 & 1.0021 & 9.7624 & 1.7440 & -1.0000 & -1.0000\end{array}$

$\begin{array}{lllllll}110 & 0.1358 & 1.8293 & 10.0425 & 1.6096 & -1.0000 & -1.0000\end{array}$

$\begin{array}{llllllll}2 & 10 & 0.0640 & 1.6974 & 11.5167 & 1.3517 & -1.0000 & -1.0000\end{array}$

$\begin{array}{llllllll}3 & 10 & 0.0846 & 1.4284 & 10.0808 & 1.8339 & -1.0000 & -1.0000\end{array}$

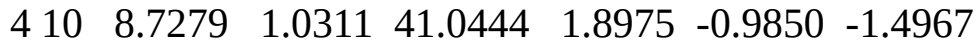

68 ! Nr of angles;at1;at2;at3;Thetao,o;ka;kb;pv1;pv2

$\begin{array}{llllllllll}1 & 1 & 1 & 59.0573 & 30.7029 & 0.7606 & 0.0000 & 0.7180 & 6.2933 & 1.1244\end{array}$

$\begin{array}{llllllllll}1 & 1 & 2 & 65.7758 & 14.5234 & 6.2481 & 0.0000 & 0.5665 & 0.0000 & 1.6255\end{array}$

$\begin{array}{llllllllll}2 & 1 & 2 & 70.2607 & 25.2202 & 3.7312 & 0.0000 & 0.0050 & 0.0000 & 2.7500\end{array}$

$\begin{array}{llllllllll}1 & 2 & 2 & 0.0000 & 0.0000 & 6.0000 & 0.0000 & 0.0000 & 0.0000 & 1.0400\end{array}$

$\begin{array}{llllllllll}1 & 2 & 1 & 0.0000 & 3.4110 & 7.7350 & 0.0000 & 0.0000 & 0.0000 & 1.0400\end{array}$

$\begin{array}{llllllllll}2 & 2 & 2 & 0.0000 & 27.9213 & 5.8635 & 0.0000 & 0.0000 & 0.0000 & 1.0400\end{array}$

$\begin{array}{llllllllll}1 & 1 & 3 & 54.7427 & 21.1992 & 1.0613 & 0.0000 & 2.9950 & 58.6562 & 1.1232\end{array}$

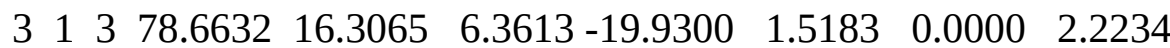

$\begin{array}{llllllllll}2 & 1 & 3 & 50.0000 & 12.9103 & 2.5311 & 0.0000 & 0.1000 & 0.0000 & 1.0000\end{array}$

$\begin{array}{llllllllll}1 & 3 & 1 & 71.6401 & 45.0000 & 1.2667 & 0.0000 & 2.8294 & 0.0000 & 1.0000\end{array}$

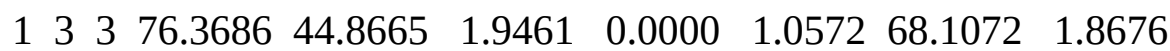

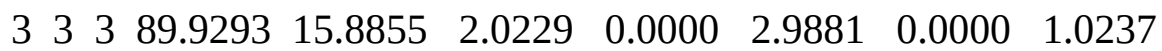

$\begin{array}{llllllllll}1 & 3 & 2 & 90.0000 & 6.6459 & 5.2255 & 0.0000 & 1.3111 & 0.0000 & 3.0000\end{array}$

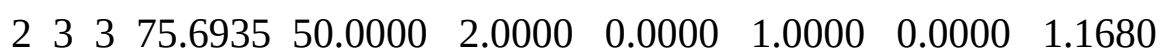

$\begin{array}{llllllllll}2 & 3 & 2 & 85.8000 & 9.8453 & 2.2720 & 0.0000 & 2.8635 & 0.0000 & 1.5800\end{array}$

$\begin{array}{llllllllll}1 & 2 & 3 & 0.0000 & 16.7302 & 1.1143 & 0.0000 & 0.0000 & 0.0000 & 1.0000\end{array}$

$\begin{array}{llllllllll}3 & 2 & 3 & 0.0000 & 15.0000 & 2.8900 & 0.0000 & 0.0000 & 0.0000 & 2.8774\end{array}$

$\begin{array}{llllllllll}2 & 2 & 3 & 0.0000 & 8.5744 & 3.0000 & 0.0000 & 0.0000 & 0.0000 & 1.0421\end{array}$

$\begin{array}{llllllllll}3 & 4 & 3 & 29.9309 & 3.6478 & 2.2492 & 0.0000 & 1.1980 & 0.0000 & 2.3810\end{array}$

$\begin{array}{llllllllll}4 & 3 & 4 & 3.3622 & 10.1592 & 2.3865 & 0.0000 & 3.2489 & 0.0000 & 2.6812\end{array}$

$\begin{array}{llllllllll}3 & 3 & 4 & 11.7872 & 50.0000 & 5.8383 & 0.0000 & 4.6977 & 0.0000 & 1.0000\end{array}$

$\begin{array}{llllllllll}3 & 4 & 4 & 15.3871 & 4.8803 & 8.3929 & 0.0000 & 4.1095 & 0.0000 & 3.4321\end{array}$

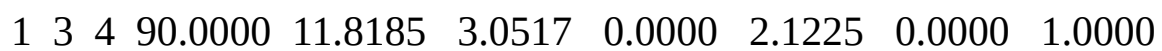

$\begin{array}{llllllllll}2 & 5 & 2 & 25.9881 & 0.0100 & 1.8827 & 0.0000 & 0.6581 & 0.0000 & 1.1500\end{array}$

$\begin{array}{llllllllll}5 & 2 & 5 & 0.0000 & 3.6249 & 1.0000 & 0.0000 & 1.0000 & 0.0000 & 1.2500\end{array}$

$\begin{array}{llllllllll}3 & 5 & 3 & 10.0000 & 0.1000 & 1.0302 & 0.0000 & 1.0000 & 0.0000 & 1.0000\end{array}$ 


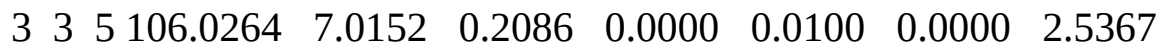

$\begin{array}{llllllllll}13 & 5 & 92.0242 & 0.1000 & 10.0000 & 0.0000 & 2.8844 & 0.0000 & 1.1706\end{array}$

$\begin{array}{llllllllll}3 & 1 & 5 & 100.2536 & 0.0100 & 4.2329 & 0.0000 & 1.7872 & 0.0000 & 2.3488\end{array}$

$\begin{array}{llllllllll}5 & 3 & 5 & 70.3878 & 3.0984 & 2.8121 & 0.0000 & 0.9139 & 0.0000 & 1.9378\end{array}$

$\begin{array}{lllllllllll}3 & 2 & 6 & 0.0000 & 1.8088 & 0.0100 & 0.0000 & 0.0000 & 0.0000 & 1.2229\end{array}$

$\begin{array}{llllllllll}2 & 6 & 2 & 0.0000 & 5.6384 & 1.3636 & 0.0000 & 0.4652 & 0.0000 & 1.0400\end{array}$

$\begin{array}{lllllllllll}6 & 4 & 6 & 19.0109 & 16.8964 & 13.2508 & 0.0000 & -0.3146 & 0.0000 & 1.3785\end{array}$

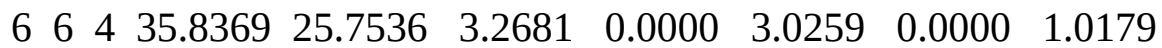

$\begin{array}{llllllllll}3 & 4 & 6 & 23.3113 & 15.3421 & 1.7515 & 0.0000 & 2.0263 & 0.0000 & 1.3581\end{array}$

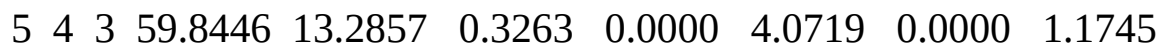

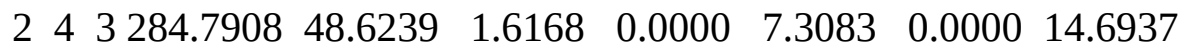

$\begin{array}{llllllllll}4 & 3 & 2 & 48.8774 & 12.4454 & 1.0604 & 0.0000 & 4.1897 & 0.0000 & 1.0077\end{array}$

$\begin{array}{llllllllll}5 & 3 & 2 & 52.6599 & 13.3819 & 1.0695 & 0.0000 & 4.3732 & 0.0000 & 1.0078\end{array}$

$\begin{array}{lllllllllll}6 & 2 & 6 & 10.0000 & 7.7335 & 1.0000 & 0.0000 & 1.2806 & 0.0000 & 1.0400\end{array}$

$\begin{array}{lllllllllll}2 & 6 & 6 & 10.0000 & 10.0000 & 3.8265 & 0.0000 & 1.0000 & 0.0000 & 1.0400\end{array}$

$\begin{array}{llllllllll}6 & 6 & 6 & 59.0573 & 30.7029 & 0.7606 & 0.0000 & 0.7180 & 6.2933 & 1.1244\end{array}$

$\begin{array}{llllllllll}2 & 2 & 6 & 10.0000 & 7.2139 & 3.7598 & 0.0000 & 1.0000 & 0.0000 & 1.7496\end{array}$

$\begin{array}{llllllllll}6 & 1 & 6 & 66.7242 & 16.7256 & 8.4462 & 0.0000 & 1.0455 & 0.0000 & 3.9255\end{array}$

$\begin{array}{llllllllll}6 & 1 & 2 & 70.0840 & 25.3540 & 3.4508 & 0.0000 & 1.0000 & 0.0000 & 3.0000\end{array}$

$\begin{array}{llllllllll}1 & 1 & 6 & 65.7758 & 14.5234 & 6.2481 & 0.0000 & 1.0000 & 0.0000 & 1.6255\end{array}$

$\begin{array}{llllllllll}16 & 6 & 10.0000 & 2.3487 & 6.0000 & 0.0000 & 5.0000 & 0.0000 & 1.0000\end{array}$

$\begin{array}{llllllllll}1 & 6 & 1 & 10.0000 & 3.4110 & 7.7350 & 0.0000 & 1.0000 & 0.0000 & 1.0400\end{array}$

$\begin{array}{llllllllll}1 & 10 & 1 & 29.1655 & 3.3035 & 0.2000 & 0.0000 & 1.1221 & 0.0000 & 1.0562\end{array}$

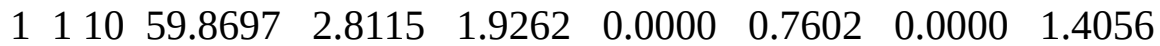

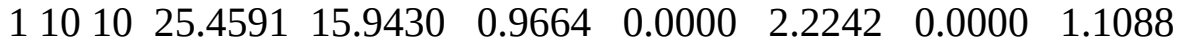

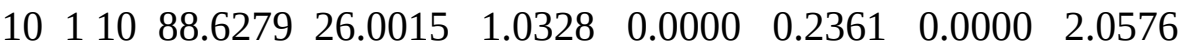

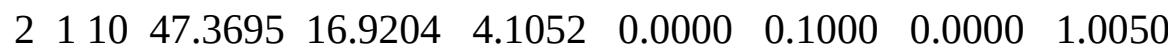

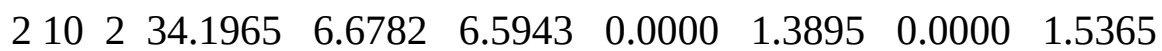

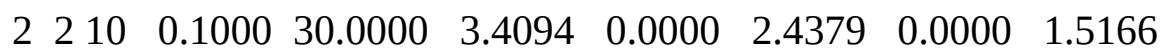

$\begin{array}{llllllllll}10 & 210 & 0.0000 & 8.2994 & 5.7832 & 0.0000 & 2.9873 & 0.0000 & 1.7716\end{array}$

$\begin{array}{lllllllll}210 & 10 & 21.2590 & 6.5954 & 0.9951 & 0.0000 & 2.8006 & 0.0000 & 1.0000\end{array}$

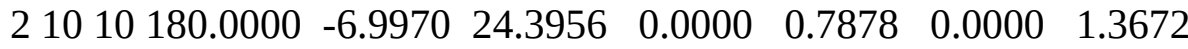

$\begin{array}{lllllllll}1310 & 90.0000 & 12.8684 & 1.4601 & 0.0000 & 0.8757 & 0.0000 & 1.0000\end{array}$

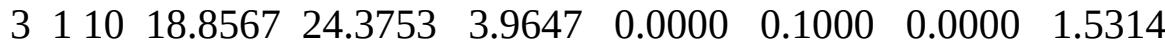

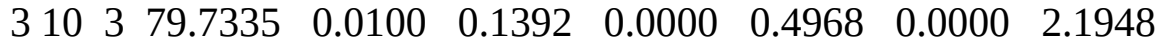

$\begin{array}{llllllllll}10 & 3 & 10 & 57.6787 & 4.8566 & 2.5768 & 0.0000 & 0.7552 & 0.0000 & 1.0000\end{array}$

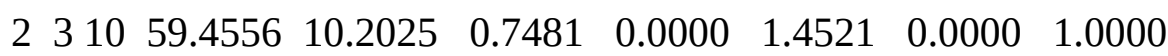

$\begin{array}{lllllllll}3 & 310 & 73.6721 & 32.6330 & 1.7223 & 0.0000 & 1.0221 & 0.0000 & 1.4351\end{array}$

$\begin{array}{lllllllll}310 & 10 & 65.7545 & 5.6268 & 4.0645 & 0.0000 & 1.7794 & 0.0000 & 2.6730\end{array}$

$\begin{array}{lllllllll}3 & 210 & 0.0000 & 4.6026 & 2.5343 & 0.0000 & 0.7284 & 0.0000 & 1.1051\end{array}$

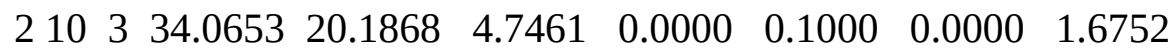

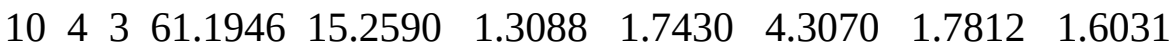

32 ! Nr of torsions;at1;at2;at3;at4;;V1;V2;V3;V2(BO);vconj;n.u;n

$\begin{array}{lllllllllll}1 & 1 & 1 & 1 & -0.2500 & 34.7453 & 0.0288 & -6.3507 & -1.6000 & 0.0000 & 0.0000\end{array}$

$\begin{array}{llllllllllll}1 & 1 & 1 & 2 & -0.2500 & 29.2131 & 0.2945 & -4.9581 & -2.1802 & 0.0000 & 0.0000\end{array}$

$\begin{array}{lllllllllll}2 & 1 & 1 & 2 & -0.2500 & 31.2081 & 0.4539 & -4.8923 & -2.2677 & 0.0000 & 0.0000\end{array}$

$\begin{array}{lllllllllll}1 & 1 & 1 & 3 & -2.5000 & 25.4016 & 1.0000 & -4.4850 & -1.1000 & 0.0000 & 0.0000\end{array}$

$\begin{array}{lllllllllll}2 & 1 & 1 & 3 & -0.9763 & 59.4161 & 1.0000 & -7.7414 & -1.0978 & 0.0000 & 0.0000\end{array}$

$\begin{array}{lllllllllll}3 & 1 & 1 & 3 & -2.5000 & 52.7614 & -1.0000 & -4.0134 & -0.8614 & 0.0000 & 0.0000\end{array}$

$\begin{array}{lllllllllll}1 & 1 & 3 & 1 & -1.9125 & 80.0000 & -1.0000 & -4.5626 & -0.9000 & 0.0000 & 0.0000\end{array}$

$\begin{array}{lllllllllll}1 & 1 & 3 & 2 & 0.6154 & 8.3019 & -0.4870 & -2.9336 & -0.9000 & 0.0000 & 0.0000\end{array}$

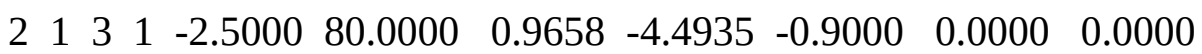




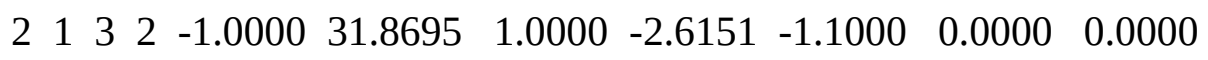

$\begin{array}{llllllllllll}1 & 1 & 3 & 3 & 0.7514 & 34.1941 & 0.5669 & -5.5360 & -2.0544 & 0.0000 & 0.0000\end{array}$

$\begin{array}{llllllllllll}2 & 1 & 3 & 3 & 2.5000 & 80.0000 & 1.0000 & -2.6841 & -2.8274 & 0.0000 & 0.0000\end{array}$

$\begin{array}{llllllllllll}3 & 1 & 3 & 1 & 0.2515 & 79.1495 & -0.6263 & -4.3647 & -3.0437 & 0.0000 & 0.0000\end{array}$

$\begin{array}{llllllllllll}3 & 1 & 3 & 2 & 1.0000 & 37.1243 & 1.0000 & -2.5000 & -3.0476 & 0.0000 & 0.0000\end{array}$

$\begin{array}{llllllllllll}3 & 1 & 3 & 3 & -1.0092 & 41.0504 & 0.3915 & -6.0913 & -2.7174 & 0.0000 & 0.0000\end{array}$

$\begin{array}{lllllllllll}1 & 3 & 3 & 1 & -1.6378 & -11.8357 & 0.3815 & -3.2104 & -2.7536 & 0.0000 & 0.0000\end{array}$

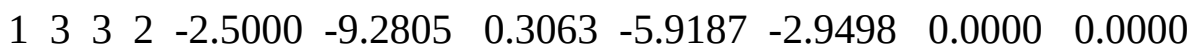

$\begin{array}{lllllllllll}2 & 3 & 3 & 2 & 0.2732 & -21.6925 & -1.0000 & -2.5000 & -0.9921 & 0.0000 & 0.0000\end{array}$

$\begin{array}{lllllllllll}1 & 3 & 3 & 3 & 2.5000 & -17.6041 & 1.0000 & -2.5000 & -0.9972 & 0.0000 & 0.0000\end{array}$

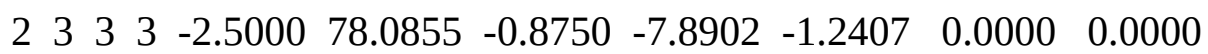

$\begin{array}{lllllllllll}3 & 3 & 3 & 3 & -2.5000 & -25.0000 & 1.0000 & -2.5000 & -0.9000 & 0.0000 & 0.0000\end{array}$

$\begin{array}{llllllllllll}0 & 1 & 2 & 0 & 0.0000 & 0.0000 & 0.0000 & 0.0000 & 0.0000 & 0.0000 & 0.0000\end{array}$

$\begin{array}{llllllllllll}0 & 2 & 2 & 0 & 0.0000 & 0.0000 & 0.0000 & 0.0000 & 0.0000 & 0.0000 & 0.0000\end{array}$

$\begin{array}{llllllllllll}0 & 2 & 3 & 0 & 0.0000 & 0.1000 & 0.0200 & -2.5415 & 0.0000 & 0.0000 & 0.0000\end{array}$

$\begin{array}{llllllllllll}0 & 1 & 1 & 0 & 0.0000 & 50.0000 & 0.3000 & -4.0000 & -2.0000 & 0.0000 & 0.0000\end{array}$

$\begin{array}{lllllllllll}0 & 3 & 3 & 0 & 0.5511 & 25.4150 & 1.1330 & -5.1903 & -1.0000 & 0.0000 & 0.0000\end{array}$

$\begin{array}{llllllllllll}0 & 1 & 6 & 0 & 3.3423 & 30.3435 & 0.0365 & -2.7171 & 0.0000 & 0.0000 & 0.0000\end{array}$

$\begin{array}{llllllllllll}0 & 6 & 6 & 0 & -0.0500 & 10.0000 & 0.1565 & -2.2006 & 0.0000 & 0.0000 & 0.0000\end{array}$

$\begin{array}{llllllllllll}0 & 2 & 6 & 0 & 0.0000 & 0.0000 & 0.0000 & 0.0000 & 0.0000 & 0.0000 & 0.0000\end{array}$

$\begin{array}{llllllllllll}1 & 1 & 1 & 6 & -0.2500 & 29.2131 & 0.2945 & -4.9581 & -2.1802 & 0.0000 & 0.0000\end{array}$

$\begin{array}{llllllllllll}6 & 1 & 1 & 6 & -0.2500 & 31.2081 & 0.4539 & -4.8923 & -2.2677 & 0.0000 & 0.0000\end{array}$

$\begin{array}{lllllllllll}6 & 6 & 6 & 6 & -0.2500 & 34.7453 & 0.0288 & -6.3507 & -1.6000 & 0.0000 & 0.0000\end{array}$

1 ! Nr of hydrogen bonds;at1;at2;at3;Rhb;Dehb;vhb1

$\begin{array}{lllllll}3 & 2 & 3 & 0.7000 & -3.5800 & 1.4500 & 19.5000\end{array}$ 


\section{$\mathrm{Ni} / \mathrm{Mn} / \mathrm{O} / \mathrm{C} / \mathrm{H}$ Force field}

Refinement of:

Ni: Supporting information for the manuscript 'Development and Validation of ReaxFF

Reactive Force Field for Hydrocarbon Chemistry Catalyzed by Nickel.', authors Jonathan E.

Mueller, Adri C. T. van Duin and William A. Goddard III

Mn: Electrode-electrolyte interface layers in lithium ion batteries using reactive force field based molecular dynamics by Sahithya Reddivari, doctoral thesis, 2016, University of Michigan

Reactive MD-force field: Mn/Ni/O/C/H Gomzi et al 2021.

39 ! Number of general parameters

50.0000 !Overcoordination parameter

9.5469 !Overcoordination parameter

1.6725 !Valency angle conjugation parameter

1.7224 !Triple bond stabilisation parameter

6.8702 !Triple bond stabilisation parameter

60.4850 !C2-correction

1.0588 !Undercoordination parameter

4.6000 !Triple bond stabilisation parameter

12.1176 !Undercoordination parameter

13.3056 !Undercoordination parameter

-55.1978 !Triple bond stabilization energy

0.0000 !Lower Taper-radius

10.0000 !Upper Taper-radius

2.8793 !Not used

33.8667 !Valency undercoordination

6.0891 !Valency angle/lone pair parameter

1.0563 !Valency angle

2.0384 !Valency angle parameter

6.1431 !Not used

6.9290 !Double bond/angle parameter

0.3989 !Double bond/angle parameter: overcoord

3.9954 !Double bond/angle parameter: overcoord

-2.4837 !Not used

5.7796 !Torsion/BO parameter

10.0000 !Torsion overcoordination

1.9487 !Torsion overcoordination

-1.2327 !Conjugation 0 (not used)

2.1645 !Conjugation

1.5591 !vdWaals shielding

0.1000 !Cutoff for bond order $(* 100)$

1.7602 !Valency angle conjugation parameter

0.6991 !Overcoordination parameter

50.0000 !Overcoordination parameter

1.8512 !Valency/lone pair parameter

0.5000 !Not used

20.0000 !Not used

5.0000 !Molecular energy (not used)

0.0000 !Molecular energy (not used)

0.7903 !Valency angle conjugation parameter

9 ! Nr of atoms; cov.r; valency;a.m;Rvdw;Evdw;gammaEEM;cov.r2; 
alfa;gammavdW;valency;Eunder;Eover;chiEEM;etaEEM;n.u. cov r3;Elp;Heat inc.;n.u.;n.u.;n.u.;n.u.

ov/un;val1;n.u.;val3,vval4

C $\quad \begin{array}{llllllll}1.3817 & 4.0000 & 12.0000 & 1.8903 & 0.1838 & 0.6387 & 1.1341 & 4.0000\end{array}$ $\begin{array}{llllllll}9.7559 & 2.1346 & 4.0000 & 34.9350 & 79.5548 & 4.9218 & 6.0000 & 0.0000\end{array}$

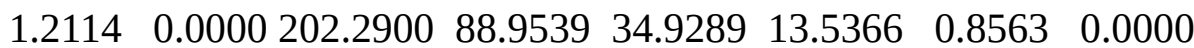
$\begin{array}{llllllll}-2.8983 & 2.5000 & 1.0560 & 44.0000 & 2.9663 & 0.0000 & 0.0000 & 0.0000\end{array}$

$\mathrm{H} \quad \begin{array}{lllllllll}\mathrm{H} & 0.8930 & 1.0000 & 1.0080 & 1.3550 & 0.0930 & 0.8203 & -0.1000 & 1.0000\end{array}$ $\begin{array}{llllllll}8.2230 & 33.2894 & 1.0000 & 0.0000 & 121.1250 & 3.7248 & 9.6093 & 1.0000\end{array}$ $\begin{array}{llllllll}-0.1000 & 0.0000 & 55.1870 & 83.0408 & 2.4197 & 0.0003 & 1.0698 & 0.0000\end{array}$ $\begin{array}{llllllll}-19.4571 & 4.2733 & 1.0330 & 81.0000 & 2.8793 & 0.0000 & 0.0000 & 0.0000\end{array}$

$\begin{array}{lllllllll}\text { O } & 1.2450 & 2.0000 & 15.9990 & 2.3890 & 0.1000 & 1.0898 & 1.0548 & 6.0000\end{array}$ $\begin{array}{llllllll}9.7300 & 13.8449 & 4.0000 & 37.5000 & 116.0768 & 8.5000 & 8.3122 & 2.0000\end{array}$ $\begin{array}{llllllll}0.9049 & 0.4056 & 68.0152 & 3.5027 & 0.7640 & 0.0021 & 0.9745 & 0.0000\end{array}$ $\begin{array}{lllllllll}-3.5500 & 2.9000 & 1.0493 & 4.0000 & 2.9225 & 0.0000 & 0.0000 & 0.0000\end{array}$

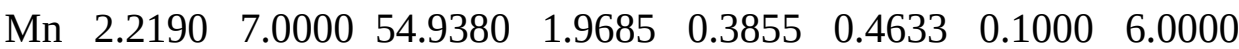
$\begin{array}{llllllll}11.2058 & 4.1928 & 4.0000 & 0.0000 & 0.0000 & -1.0000 & 6.1911 & 0.0000\end{array}$ $\begin{array}{llllllll}0.1000 & 0.0000 & 152.6300 & 3.4529 & 0.0722 & 3.1767 & 0.8563 & 0.0000\end{array}$ $\begin{array}{lllllllll}-25.0000 & 3.1072 & 1.0338 & 8.0000 & 3.4590 & 0.0000 & 0.0000 & 0.0000\end{array}$

$\begin{array}{lllllllll}\mathrm{Li} & 1.9814 & 1.0000 & 6.9410 & 1.8000 & 0.2939 & 0.9387 & -0.1000 & 1.0000\end{array}$ $\begin{array}{llllllll}9.0616 & 1.3258 & 1.0000 & 0.0000 & 0.0000 & -3.0000 & 10.0241 & 0.0000\end{array}$ $\begin{array}{llllllll}-1.0000 & 0.0000 & 37.5000 & 5.4409 & 6.9107 & 0.1973 & 0.8563 & 0.0000\end{array}$ $\begin{array}{llllllll}-2.5068 & 2.2989 & 1.0338 & 1.0000 & 2.8103 & 1.3000 & 0.2000 & 13.0000\end{array}$

$\begin{array}{lllllllll}\text { F } & 1.7938 & 1.0000 & 18.9984 & 1.4139 & 0.3134 & 0.7750 & -0.1000 & 7.0000\end{array}$ $\begin{array}{llllllll}10.3051 & 15.1397 & 1.0000 & 9.2533 & 0.2000 & 9.8105 & 8.6941 & 0.0000\end{array}$ $\begin{array}{llllllll}-1.0000 & 3.5571 & 18.0000 & 6.9821 & 4.1799 & 1.0561 & 0.0000 & 0.0000\end{array}$ $\begin{array}{llllllll}-6.3417 & 2.6656 & 1.0493 & 4.0000 & 2.9225 & 0.0000 & 0.0000 & 0.0000\end{array}$

$\begin{array}{lllllllll}\mathrm{P} & 1.5994 & 3.0000 & 30.9738 & 1.7000 & 0.1743 & 1.0385 & 1.3000 & 5.0000\end{array}$ $\begin{array}{llllllll}9.1909 & 14.2932 & 5.0000 & 0.0000 & 0.0000 & 0.9528 & 7.9121 & 0.0000\end{array}$ $\begin{array}{llllllll}-1.0000 & 10.2596 & 1.5000 & 0.2205 & 16.7429 & 15.9629 & 0.0000 & 0.0000\end{array}$ $\begin{array}{llllllll}-2.5000 & 1.6114 & 1.0338 & 5.0000 & 2.8793 & 0.0000 & 0.0000 & 0.0000\end{array}$

$\begin{array}{lllllllll}\text { Ni } & 1.8201 & 2.0000 & 58.6900 & 1.9449 & 0.1880 & 0.8218 & 0.1000 & 2.0000\end{array}$ $\begin{array}{llllllll}12.1594 & 3.8387 & 2.0000 & 0.0000 & 0.0000 & 4.8038 & 7.3852 & 0.0000\end{array}$ $\begin{array}{llllllll}-1.0000 & 0.0000 & 95.6300 & 50.6786 & 0.6762 & 0.0981 & 0.8563 & 0.0000\end{array}$ $\begin{array}{lllllllll}-3.7733 & 3.6035 & 1.0338 & 8.0000 & 2.5791 & 0.0000 & 0.0000 & 0.0000\end{array}$

$\begin{array}{lllllllll}\mathrm{Al} & -0.1000 & 2.0000 & 1.0080 & 2.0000 & 0.0000 & 1.0000 & -0.1000 & 6.0000\end{array}$ $\begin{array}{llllllll}10.0000 & 2.5000 & 4.0000 & 0.0000 & 0.0000 & 8.5000 & 1.5000 & 0.0000\end{array}$ $\begin{array}{llllllll}-0.1000 & 0.0000 & -2.3700 & 8.7410 & 13.3640 & 0.6690 & 0.9745 & 0.0000\end{array}$ $\begin{array}{llllllll}-11.0000 & 2.7466 & 1.0338 & 6.2998 & 2.8793 & 0.0000 & 0.0000 & 0.0000\end{array}$

$33 \quad$ ! Nr of bonds; Edis1;LPpen;n.u.;pbe1;pbo5;13corr;pbo6 pbe2;pbo3;pbo4;Etrip;pbo1;pbo2;ovcorr

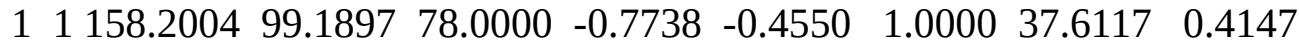
$\begin{array}{llllllll}0.4590 & -0.1000 & 9.1628 & 1.0000 & -0.0777 & 6.7268 & 1.0000 & 0.0000\end{array}$

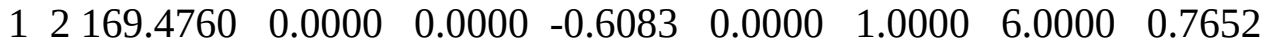
$\begin{array}{llllllll}5.2290 & 1.0000 & 0.0000 & 1.0000 & -0.0553 & 6.9316 & 0.0000 & 0.0000\end{array}$

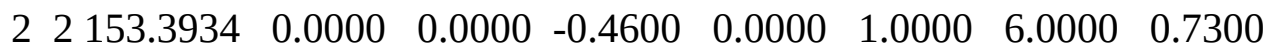
$\begin{array}{llllllll}6.2500 & 1.0000 & 0.0000 & 1.0000 & -0.0790 & 6.0552 & 0.0000 & 0.0000\end{array}$

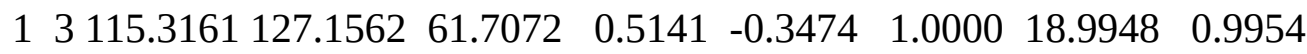
$\begin{array}{llllllll}1.5618 & -0.3414 & 8.9489 & 1.0000 & -0.1628 & 5.6821 & 0.0000 & 0.0000\end{array}$

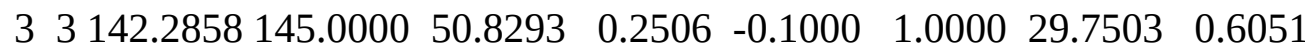
$\begin{array}{llllllll}0.3451 & -0.1055 & 9.0000 & 1.0000 & -0.1225 & 5.5000 & 1.0000 & 0.0000\end{array}$

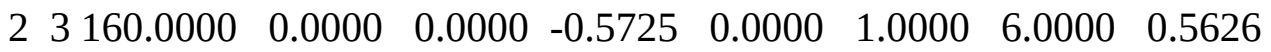


$\begin{array}{llllllll}1.1150 & 1.0000 & 0.0000 & 0.0000 & -0.0920 & 4.2790 & 0.0000 & 0.0000\end{array}$

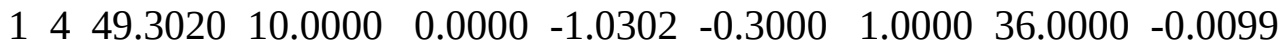
$\begin{array}{llllllll}1.8910 & -0.4204 & 7.6871 & 1.0000 & -0.0880 & 6.6676 & 1.0000 & 0.0000\end{array}$

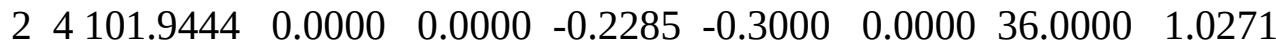
$\begin{array}{llllllll}7.0832 & -0.1532 & 19.8594 & 1.0000 & -0.0836 & 4.7210 & 0.0000 & 0.0000\end{array}$

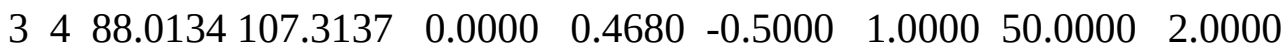
$\begin{array}{llllllll}7.5815 & -0.4509 & 7.6953 & 1.0000 & -0.0952 & 5.1424 & 1.0000 & 0.0000\end{array}$

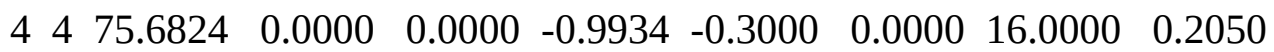
$\begin{array}{llllllll}2.2857 & -0.3000 & 16.0000 & 1.0000 & -0.1230 & 8.7005 & 0.0000 & 0.0000\end{array}$

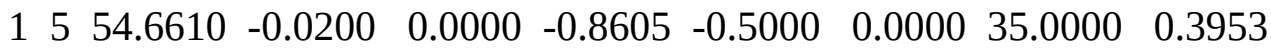
$\begin{array}{llllllll}0.6908 & -0.2500 & 11.9965 & 1.0000 & -0.0668 & 9.0596 & 0.0000 & 0.0000\end{array}$

$\begin{array}{llllllllll}2 & 5 & 59.2034 & 0.0000 & 0.0000 & 0.1240 & 0.0000 & 0.0000 & 6.0000 & 0.4000\end{array}$ $\begin{array}{llllllll}1.0000 & 0.0000 & 12.0000 & 1.0000 & -0.0565 & 4.9575 & 0.0000 & 0.0000\end{array}$

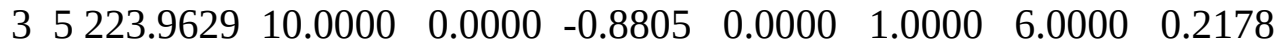
$\begin{array}{llllllll}4.2326 & -0.1048 & 7.0167 & 1.0000 & -0.0867 & 4.2825 & 0.0000 & 0.0000\end{array}$

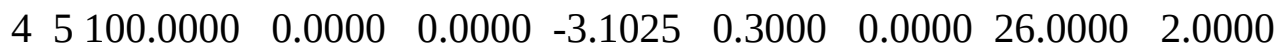
$\begin{array}{llllllll}3.2823 & 0.0000 & 12.0000 & 1.0000 & -0.0800 & 6.9752 & 0.0000 & 0.0000\end{array}$

$\begin{array}{llllllllll}5 & 5 & 34.3154 & 0.0000 & 0.0000 & 0.5995 & 0.3000 & 0.0000 & 26.0000 & 0.5445\end{array}$ $\begin{array}{llllllll}0.5752 & 0.0000 & 12.0000 & 1.0000 & -0.1382 & 4.5000 & 0.0000 & 0.0000\end{array}$

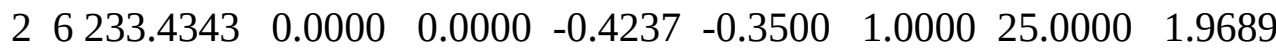
$\begin{array}{llllllll}4.7870 & -0.2500 & 15.0000 & 1.0000 & -0.1288 & 4.9159 & 1.0000 & 0.0000\end{array}$

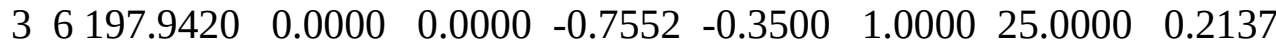
$\begin{array}{llllllll}9.5888 & -0.2500 & 15.0000 & 1.0000 & -0.1477 & 7.8947 & 1.0000 & 0.0000\end{array}$

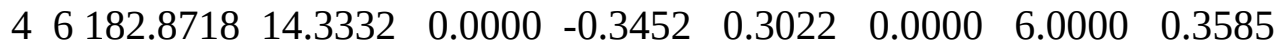
$\begin{array}{llllllll}2.6684 & -0.1484 & 11.8165 & 1.0000 & -0.0940 & 5.8491 & 0.0000 & 0.0000\end{array}$

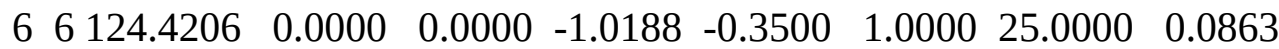
$\begin{array}{llllllll}8.4257 & -0.2500 & 15.0000 & 1.0000 & -0.2337 & 8.1452 & 1.0000 & 0.0000\end{array}$

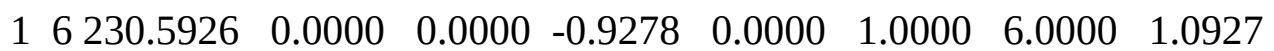
$\begin{array}{llllllll}7.1644 & 1.0000 & 0.0000 & 1.0000 & -0.0732 & 5.9745 & 0.0000 & 0.0000\end{array}$

$\begin{array}{llllllllll}5 & 6 & 92.6032 & 0.0000 & 0.0000 & -0.5454 & -0.5582 & 0.0000 & 44.9378 & 0.3238\end{array}$ $\begin{array}{llllllll}1.0563 & -0.2500 & 15.0000 & 1.0000 & -0.1206 & 4.0000 & 0.0000 & 0.0000\end{array}$

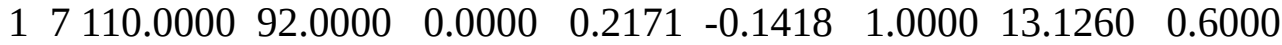
$\begin{array}{llllllll}0.3601 & -0.1310 & 10.7257 & 1.0000 & -0.0869 & 5.3302 & 1.0000 & 0.0000\end{array}$

$\begin{array}{llllllllll}2 & 7 & 0.1466 & 0.0000 & 0.0000 & 0.2250 & -0.1418 & 1.0000 & 13.1260 & 0.6000\end{array}$ $\begin{array}{llllllll}0.3912 & -0.1310 & 0.0000 & 1.0000 & -0.1029 & 9.3302 & 0.0000 & 0.0000\end{array}$

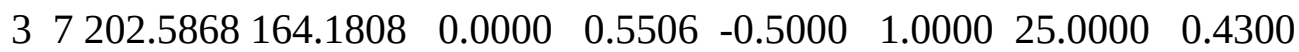

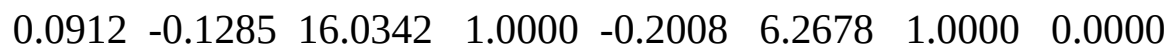

$\begin{array}{llllllllll}7 & 7 & 0.0000 & 0.0000 & 0.0000 & 0.2171 & -0.5000 & 1.0000 & 35.0000 & 0.6000\end{array}$ $\begin{array}{llllllll}0.5000 & -0.5000 & 20.0000 & 1.0000 & -0.2000 & 10.0000 & 1.0000 & 0.0000\end{array}$

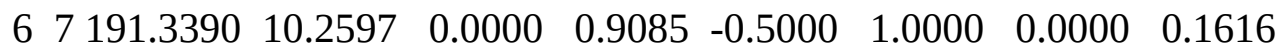
$\begin{array}{llllllll}0.0940 & -0.1039 & 10.0002 & 1.0000 & -0.1073 & 9.9960 & 1.0000 & 0.0000\end{array}$

$\begin{array}{llllllllll}5 & 7 & 0.0000 & 0.0000 & 0.0000 & 0.5000 & -0.2000 & 0.0000 & 16.0000 & 0.5000\end{array}$ $\begin{array}{llllllll}1.0001 & -0.2000 & 15.0000 & 1.0000 & -0.1000 & 10.0000 & 0.0000 & 0.0000\end{array}$

$\begin{array}{llllllllll}4 & 7 & 0.0000 & 0.0000 & 0.0000 & 0.5000 & -0.2000 & 0.0000 & 16.0000 & 0.5000\end{array}$ $\begin{array}{llllllll}1.0001 & -0.2000 & 15.0000 & 1.0000 & -0.1000 & 10.0000 & 0.0000 & 0.0000\end{array}$

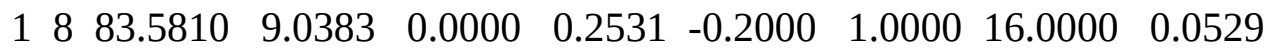

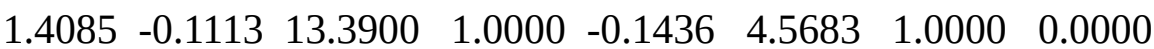

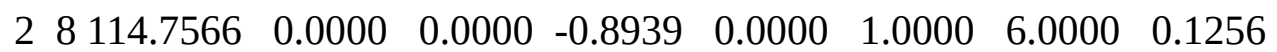
$\begin{array}{llllllll}0.1054 & 1.0000 & 0.0000 & 1.0000 & -0.1196 & 5.0815 & 0.0000 & 0.0000\end{array}$

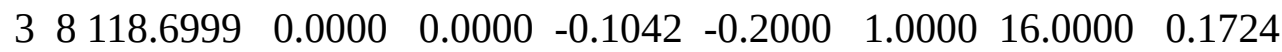
$\begin{array}{llllllll}0.8280 & -0.2500 & 15.0000 & 1.0000 & -0.1013 & 5.6326 & 1.0000 & 0.0000\end{array}$

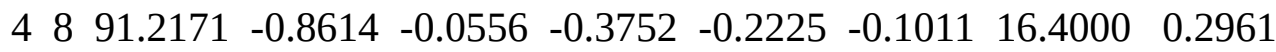


$\begin{array}{llllllll}1.2251 & -0.2172 & 15.3527 & 1.0268 & -0.1354 & 4.2875 & 0.0348 & -0.0200\end{array}$

$\begin{array}{llllllllll}8 & 8 & 91.2220 & 0.0000 & 0.0000 & -0.2538 & -0.2000 & 0.0000 & 16.0000 & 0.2688\end{array}$

$\begin{array}{llllllll}1.4651 & -0.2000 & 15.0000 & 1.0000 & -0.1435 & 4.3908 & 0.0000 & 0.0000\end{array}$

20 ! Nr of off-diagonal terms; Ediss;Ro;gamma;rsigma;rpi;rpi2

$\begin{array}{llllllll}1 & 2 & 0.1239 & 1.4004 & 9.8467 & 1.1210 & -1.0000 & -1.0000\end{array}$

$\begin{array}{llllllll}2 & 3 & 0.0283 & 1.2885 & 10.9190 & 0.9215 & -1.0000 & -1.0000\end{array}$

$\begin{array}{llllllll}1 & 3 & 0.0503 & 1.8006 & 10.2114 & 1.3492 & 1.1992 & 1.0506\end{array}$

$\begin{array}{llllllll}1 & 4 & 0.0475 & 1.6810 & 8.8902 & 1.5153 & 1.0000 & -1.0000\end{array}$

$\begin{array}{llllllll}2 & 4 & 0.1017 & 1.3259 & 12.5406 & 1.4534 & -1.0000 & -1.0000\end{array}$

$\begin{array}{llllllll}3 & 4 & 0.1121 & 2.0879 & 11.2110 & 1.5063 & 1.4795 & -1.0000\end{array}$

$\begin{array}{llllllll}1 & 5 & 0.0270 & 2.4124 & 11.4640 & 1.7840 & 1.0000 & 1.0000\end{array}$

$\begin{array}{llllllll}2 & 5 & 0.1149 & 1.4658 & 11.0886 & 1.3337 & -1.0000 & -1.0000\end{array}$

$\begin{array}{llllllll}3 & 5 & 0.0687 & 1.8765 & 12.0451 & 1.2928 & -1.0000 & -1.0000\end{array}$

$\begin{array}{llllllll}4 & 5 & 7.1011 & 0.9961 & 40.6852 & 1.7459 & -1.0000 & -1.0000\end{array}$

$\begin{array}{llllllll}2 & 6 & 0.1087 & 1.8734 & 9.1166 & 1.0000 & -1.0000 & -1.0000\end{array}$

$\begin{array}{llllllll}3 & 6 & 0.1714 & 1.6576 & 10.7298 & 1.5553 & -1.0000 & -1.0000\end{array}$

$\begin{array}{llllllll}4 & 6 & 0.0837 & 1.9512 & 12.2762 & 1.4795 & -1.0000 & -1.0000\end{array}$

$\begin{array}{llllllll}5 & 6 & 0.1092 & 1.7165 & 10.1139 & 1.5274 & -1.0000 & -1.0000\end{array}$

$\begin{array}{llllllll}1 & 6 & 0.1485 & 1.3609 & 11.8373 & 1.3335 & 1.4000 & -1.0000\end{array}$

$\begin{array}{llllllll}6 & 7 & 0.1435 & 1.0021 & 9.7624 & 1.7440 & -1.0000 & -1.0000\end{array}$

$\begin{array}{llllllll}1 & 8 & 0.0800 & 1.7085 & 10.0895 & 1.5504 & 1.4005 & -1.0000\end{array}$

$\begin{array}{llllllll}2 & 8 & 0.0366 & 1.7306 & 11.1019 & 1.2270 & -1.0000 & -1.0000\end{array}$

$\begin{array}{llllllll}3 & 8 & 0.0504 & 1.7959 & 11.7893 & 1.4423 & -1.0000 & -1.0000\end{array}$

$\begin{array}{llllllll}4 & 8 & 8.6568 & 1.1322 & 40.5350 & 2.0000 & -0.9850 & -1.5967\end{array}$

70 ! Nr of angles;at1;at2;at3;Thetao,o;ka;kb;pv1;pv2

$\begin{array}{llllllllll}1 & 1 & 1 & 59.0573 & 30.7029 & 0.7606 & 0.0000 & 0.7180 & 6.2933 & 1.1244\end{array}$

$\begin{array}{llllllllll}1 & 1 & 2 & 65.7758 & 14.5234 & 6.2481 & 0.0000 & 0.5665 & 0.0000 & 1.6255\end{array}$

$\begin{array}{llllllllll}2 & 1 & 2 & 70.2607 & 25.2202 & 3.7312 & 0.0000 & 0.0050 & 0.0000 & 2.7500\end{array}$

$\begin{array}{llllllllll}1 & 2 & 2 & 0.0000 & 0.0000 & 6.0000 & 0.0000 & 0.0000 & 0.0000 & 1.0400\end{array}$

$\begin{array}{llllllllll}1 & 2 & 1 & 0.0000 & 3.4110 & 7.7350 & 0.0000 & 0.0000 & 0.0000 & 1.0400\end{array}$

$\begin{array}{llllllllll}2 & 2 & 2 & 0.0000 & 27.9213 & 5.8635 & 0.0000 & 0.0000 & 0.0000 & 1.0400\end{array}$

$\begin{array}{llllllllll}1 & 1 & 3 & 54.7427 & 21.1992 & 1.0613 & 0.0000 & 2.9950 & 58.6562 & 1.1232\end{array}$

$\begin{array}{llllllllll}3 & 1 & 3 & 78.6632 & 16.3065 & 6.3613 & -19.9300 & 1.5183 & 0.0000 & 2.2234\end{array}$

$\begin{array}{llllllllll}2 & 1 & 3 & 50.0000 & 12.9103 & 2.5311 & 0.0000 & 0.1000 & 0.0000 & 1.0000\end{array}$

$\begin{array}{llllllllll}1 & 3 & 1 & 71.6401 & 45.0000 & 1.2667 & 0.0000 & 2.8294 & 0.0000 & 1.0000\end{array}$

$\begin{array}{llllllllll}1 & 3 & 3 & 76.3686 & 44.8665 & 1.9461 & 0.0000 & 1.0572 & 68.1072 & 1.8676\end{array}$

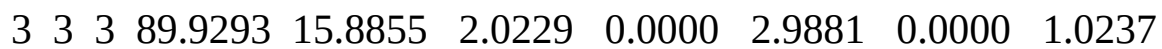

$\begin{array}{llllllllll}1 & 3 & 2 & 90.0000 & 6.6459 & 5.2255 & 0.0000 & 1.3111 & 0.0000 & 3.0000\end{array}$

$\begin{array}{llllllllll}2 & 3 & 3 & 75.6935 & 50.0000 & 2.0000 & 0.0000 & 1.0000 & 0.0000 & 1.1680\end{array}$

$\begin{array}{llllllllll}2 & 3 & 2 & 85.8000 & 9.8453 & 2.2720 & 0.0000 & 2.8635 & 0.0000 & 1.5800\end{array}$

$\begin{array}{llllllllll}1 & 2 & 3 & 0.0000 & 16.7302 & 1.1143 & 0.0000 & 0.0000 & 0.0000 & 1.0000\end{array}$

$\begin{array}{llllllllll}3 & 2 & 3 & 0.0000 & 15.0000 & 2.8900 & 0.0000 & 0.0000 & 0.0000 & 2.8774\end{array}$

$\begin{array}{llllllllll}2 & 2 & 3 & 0.0000 & 8.5744 & 3.0000 & 0.0000 & 0.0000 & 0.0000 & 1.0421\end{array}$

$\begin{array}{llllllllll}3 & 4 & 3 & 29.9309 & 3.6478 & 2.2492 & 0.0000 & 1.1980 & 0.0000 & 2.3810\end{array}$

$\begin{array}{llllllllll}4 & 3 & 4 & 3.3622 & 10.1592 & 2.3865 & 0.0000 & 3.2489 & 0.0000 & 2.6812\end{array}$

$\begin{array}{llllllllll}3 & 3 & 4 & 11.7872 & 50.0000 & 5.8383 & 0.0000 & 4.6977 & 0.0000 & 1.0000\end{array}$

$\begin{array}{llllllllll}3 & 4 & 4 & 15.3871 & 4.8803 & 8.3929 & 0.0000 & 4.1095 & 0.0000 & 3.4321\end{array}$

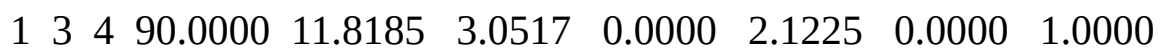

$\begin{array}{llllllllll}2 & 5 & 2 & 25.9881 & 0.0100 & 1.8827 & 0.0000 & 0.6581 & 0.0000 & 1.1500\end{array}$

$\begin{array}{llllllllll}5 & 2 & 5 & 0.0000 & 3.6249 & 1.0000 & 0.0000 & 1.0000 & 0.0000 & 1.2500\end{array}$

$\begin{array}{llllllllll}3 & 5 & 3 & 10.0000 & 0.1000 & 1.0302 & 0.0000 & 1.0000 & 0.0000 & 1.0000\end{array}$

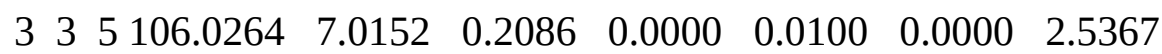


$\begin{array}{llllllllll}1 & 3 & 5 & 92.0242 & 0.1000 & 10.0000 & 0.0000 & 2.8844 & 0.0000 & 1.1706\end{array}$

$\begin{array}{llllllllll}3 & 1 & 5 & 100.2536 & 0.0100 & 4.2329 & 0.0000 & 1.7872 & 0.0000 & 2.3488\end{array}$

$\begin{array}{llllllllll}5 & 3 & 5 & 70.3878 & 3.0984 & 2.8121 & 0.0000 & 0.9139 & 0.0000 & 1.9378\end{array}$

$\begin{array}{llllllllll}3 & 2 & 6 & 0.0000 & 1.8088 & 0.0100 & 0.0000 & 0.0000 & 0.0000 & 1.2229\end{array}$

$\begin{array}{llllllllll}2 & 6 & 2 & 0.0000 & 5.6384 & 1.3636 & 0.0000 & 0.4652 & 0.0000 & 1.0400\end{array}$

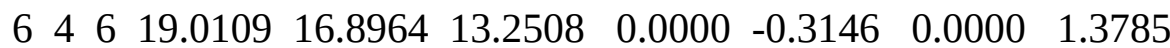

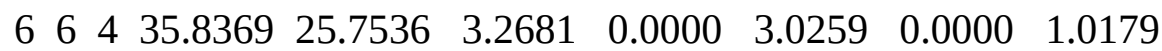

$\begin{array}{llllllllll}3 & 4 & 6 & 23.3113 & 15.3421 & 1.7515 & 0.0000 & 2.0263 & 0.0000 & 1.3581\end{array}$

$\begin{array}{llllllllll}5 & 4 & 3 & 59.8446 & 13.2857 & 0.3263 & 0.0000 & 4.0719 & 0.0000 & 1.1745\end{array}$

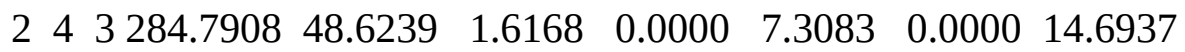

$\begin{array}{llllllllll}4 & 3 & 2 & 48.8774 & 12.4454 & 1.0604 & 0.0000 & 4.1897 & 0.0000 & 1.0077\end{array}$

$\begin{array}{llllllllll}5 & 3 & 2 & 52.6599 & 13.3819 & 1.0695 & 0.0000 & 4.3732 & 0.0000 & 1.0078\end{array}$

$\begin{array}{llllllllll}6 & 2 & 6 & 10.0000 & 7.7335 & 1.0000 & 0.0000 & 1.2806 & 0.0000 & 1.0400\end{array}$

$\begin{array}{llllllllll}2 & 6 & 6 & 10.0000 & 10.0000 & 3.8265 & 0.0000 & 1.0000 & 0.0000 & 1.0400\end{array}$

$\begin{array}{llllllllll}6 & 6 & 6 & 59.0573 & 30.7029 & 0.7606 & 0.0000 & 0.7180 & 6.2933 & 1.1244\end{array}$

$\begin{array}{llllllllll}2 & 2 & 6 & 10.0000 & 7.2139 & 3.7598 & 0.0000 & 1.0000 & 0.0000 & 1.7496\end{array}$

$\begin{array}{llllllllll}6 & 1 & 6 & 66.7242 & 16.7256 & 8.4462 & 0.0000 & 1.0455 & 0.0000 & 3.9255\end{array}$

$\begin{array}{llllllllll}6 & 1 & 2 & 70.0840 & 25.3540 & 3.4508 & 0.0000 & 1.0000 & 0.0000 & 3.0000\end{array}$

$\begin{array}{llllllllll}1 & 1 & 6 & 65.7758 & 14.5234 & 6.2481 & 0.0000 & 1.0000 & 0.0000 & 1.6255\end{array}$

$\begin{array}{llllllllll}1 & 6 & 6 & 10.0000 & 2.3487 & 6.0000 & 0.0000 & 5.0000 & 0.0000 & 1.0000\end{array}$

$\begin{array}{llllllllll}1 & 6 & 1 & 10.0000 & 3.4110 & 7.7350 & 0.0000 & 1.0000 & 0.0000 & 1.0400\end{array}$

$\begin{array}{llllllllll}1 & 8 & 1 & 62.5000 & 16.6806 & 0.7981 & 0.0000 & 0.9630 & 0.0000 & 1.0711\end{array}$

$\begin{array}{llllllllll}1 & 1 & 8 & 87.6241 & 12.6504 & 1.8145 & 0.0000 & 0.6154 & 0.0000 & 1.5298\end{array}$

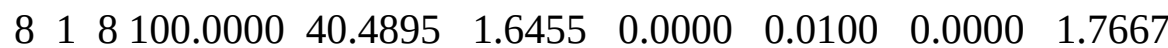

$\begin{array}{llllllllll}1 & 8 & 8 & 5.0994 & 3.1824 & 0.7016 & 0.0000 & 0.7465 & 0.0000 & 2.2665\end{array}$

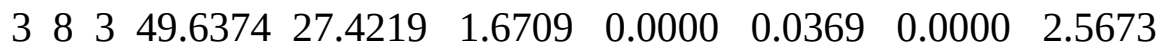

$\begin{array}{llllllllll}3 & 3 & 8 & 90.0000 & 31.1442 & 5.0000 & 0.0000 & 0.5686 & 0.0000 & 2.0931\end{array}$

$\begin{array}{llllllllll}8 & 3 & 8 & 42.4507 & 5.2354 & 0.5469 & 0.0000 & 1.5478 & 0.0000 & 1.0400\end{array}$

$\begin{array}{llllllllll}3 & 8 & 8 & 39.2561 & 2.4303 & 3.7193 & 0.0000 & 0.8924 & 0.0000 & 1.9950\end{array}$

$\begin{array}{llllllllll}2 & 8 & 2 & 106.3969 & 30.0000 & 0.9614 & 0.0000 & 1.9664 & 0.0000 & 2.2693\end{array}$

$\begin{array}{llllllllll}2 & 2 & 8 & 0.0000 & 26.3327 & 4.6867 & 0.0000 & 0.8177 & 0.0000 & 1.0404\end{array}$

$\begin{array}{llllllllll}8 & 2 & 8 & 0.0000 & 60.0000 & 1.8471 & 0.0000 & 0.6331 & 0.0000 & 1.8931\end{array}$

$\begin{array}{llllllllll}2 & 8 & 8 & 30.3748 & 1.0000 & 4.8528 & 0.0000 & 0.1019 & 0.0000 & 3.1660\end{array}$

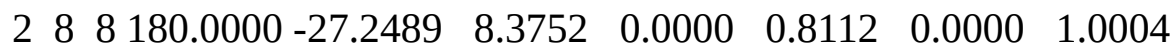

$\begin{array}{llllllllll}1 & 8 & 2 & 97.5742 & 10.9373 & 2.5200 & 0.0000 & 1.8558 & 0.0000 & 1.0000\end{array}$

$\begin{array}{llllllllll}1 & 2 & 8 & 0.0000 & 0.2811 & 1.1741 & 0.0000 & 0.9136 & 0.0000 & 3.8138\end{array}$

$\begin{array}{llllllllll}2 & 1 & 8 & 84.0006 & 45.0000 & 0.6271 & 0.0000 & 3.0000 & 0.0000 & 1.0000\end{array}$

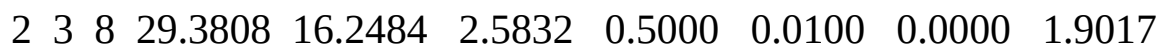

$\begin{array}{llllllllll}1 & 8 & 3 & 70.0000 & 25.0000 & 1.0000 & 0.0000 & 1.0000 & 0.0000 & 1.2500\end{array}$

$\begin{array}{llllllllll}1 & 3 & 8 & 70.0000 & 25.0000 & 1.0000 & 0.0000 & 1.0000 & 0.0000 & 1.2500\end{array}$

$\begin{array}{llllllllll}3 & 1 & 8 & 70.0000 & 25.0000 & 1.0000 & 0.0000 & 1.0000 & 0.0000 & 1.2500\end{array}$

$\begin{array}{llllllllll}3 & 2 & 8 & 0.0000 & 7.1233 & 1.9895 & 0.5000 & 0.3233 & 0.0000 & 1.1000\end{array}$

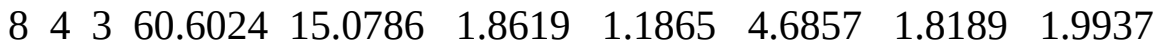

39 ! Nr of torsions;at1;at2;at3;at4;:V1;V2;V3;V2(BO);vconj;n.u;n

$\begin{array}{llllllllllll}1 & 1 & 1 & 1 & -0.2500 & 34.7453 & 0.0288 & -6.3507 & -1.6000 & 0.0000 & 0.0000\end{array}$

$\begin{array}{lllllllllll}1 & 1 & 1 & 2 & -0.2500 & 29.2131 & 0.2945 & -4.9581 & -2.1802 & 0.0000 & 0.0000\end{array}$

$\begin{array}{lllllllllll}2 & 1 & 1 & 2 & -0.2500 & 31.2081 & 0.4539 & -4.8923 & -2.2677 & 0.0000 & 0.0000\end{array}$

$\begin{array}{lllllllllll}1 & 1 & 1 & 3 & -2.5000 & 25.4016 & 1.0000 & -4.4850 & -1.1000 & 0.0000 & 0.0000\end{array}$

$\begin{array}{llllllllllll}2 & 1 & 1 & 3 & -0.9763 & 59.4161 & 1.0000 & -7.7414 & -1.0978 & 0.0000 & 0.0000\end{array}$

$\begin{array}{lllllllllll}3 & 1 & 1 & 3 & -2.5000 & 52.7614 & -1.0000 & -4.0134 & -0.8614 & 0.0000 & 0.0000\end{array}$

$\begin{array}{lllllllllll}1 & 1 & 3 & 1 & -1.9125 & 80.0000 & -1.0000 & -4.5626 & -0.9000 & 0.0000 & 0.0000\end{array}$

$\begin{array}{lllllllllll}1 & 1 & 3 & 2 & 0.6154 & 8.3019 & -0.4870 & -2.9336 & -0.9000 & 0.0000 & 0.0000\end{array}$ 


\begin{tabular}{|c|c|c|c|c|c|}
\hline \\
\hline & & $000-2$ & & & \\
\hline 3 & 0.751434 .1941 & $0.5669-5.5360$ & -2.0544 & 0.0000 & 0000 \\
\hline 3 & 2.500080 .0000 & $1.0000-2.6841$ & -2.8274 & 0.0000 & 000 \\
\hline 3 & 0.251579 .1495 & $-0.6263-4.3647$ & -3.0437 & 0.0000 & 0.000 \\
\hline & 1.000037 .1 & $1.0000-2.5000$ & -3.0476 & 0.0000 & 000 \\
\hline & -1.009241 .0504 & $0.3915-6.0913$ & -2.7174 & 0.0000 & 0.000 \\
\hline & $78-11.8357$ & $0.3815-3.2104$ & -2.7536 & 0.0000 & 0.0000 \\
\hline & $00-9$. & $0.3063 \quad-5.91$ & 498 & 0.0000 & 0.0000 \\
\hline & 0.2 & $-1.0000-2$ & -( & 00 & 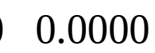 \\
\hline & 41 & $1.0000-2$. & -0 & 0.0 & 0 . \\
\hline & 0078 & $-0.8750-7.8902$ & -1 & 0.0000 & 0 . \\
\hline & -2.5 & $1.0000-2.5$ & -0 & 0.0 & \\
\hline & $0.0000 \quad 0$. & $0.0000 \quad 0.0$ & & 0.0 & \\
\hline & $0.0000 \quad 0.0$ & $0.0000 \quad 0.0$ & & & \\
\hline & $0.0000 \quad 0.1000$ & $0.0200-2.5415$ & 0.0000 & 0.00 & 0.0 \\
\hline & 0.000050 & $0.3000-4$ & -2 & 0. & \\
\hline & 1125. & $1.1330-5$. & -1.0000 & 00 & 00 \\
\hline & 2330. & $0.0365-2.7171$ & 00 & 0.0 & 00 \\
\hline & -0.050010 .0000 & $0.1565-2.2006$ & 0.0000 & 0.0000 & 0. \\
\hline & $0.0000 \quad 0.0000$ & $0.0000 \quad 0.0000$ & 0.0000 & 0.00 & 0.0 \\
\hline & -0.250029 & $0.2945-4.9$ & -2 & 0.0000 & 0.0000 \\
\hline & $-0.2500 \quad 31.2081$ & $0.4539-4.8923$ & -2.2677 & 0.0000 & 0.000 \\
\hline & -0.250034 & $0.0288-6$. & -1 & 00 & 0 . \\
\hline & $0.0000 \quad 5.0$ & $0.4000-6.0$ & $0 .($ & 0.0 & 0.0 \\
\hline & 0.000044 .3024 & $0.4000-4.0000$ & 0.0000 & 0.0 & 00 \\
\hline & 0.000021 .7038 & $0.0100-4.0000$ & 0.0000 & 0.0000 & 0.0000 \\
\hline & $0.0000 \quad 5.2$ & $0.0100-6.0$ & 0.0 & 0.0 & \\
\hline & $0.0000 \quad 5.1676$ & $0.0100-5.9539$ & 0.0000 & 0.0000 & 0.0000 \\
\hline & $0.0000 \quad 5.1676$ & $0.0100-5.9539$ & 0.0000 & 0.0000 & 0.0000 \\
\hline & 0.050930 .0000 & $0.5000-4.0000$ & 0.0000 & 0.0000 & 0.00 \\
\hline \multicolumn{6}{|c|}{ ! Nr of hydrogen bonds;at1;at2;at3;Rhb;Dehb;vhb1 } \\
\hline \multicolumn{6}{|c|}{ 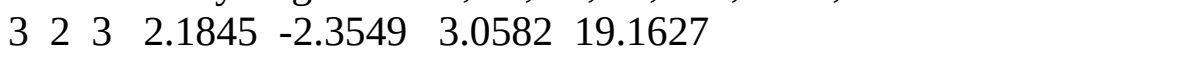 } \\
\hline \multicolumn{6}{|l|}{$3:$} \\
\hline & $38-3.5421$ & 82 & & & \\
\hline & & & & & \\
\hline
\end{tabular}

